COMMUNICATIONS IN

ANALYSIS AND GEOMETRY

Volume 11, Number 5, 945-986, 2003

\title{
Interior Regularity of Solutions to the Isotropically Constrained Plateau Problem
}

\begin{abstract}
WEIYANG QIU
In this paper, we study the regularity of isotropically areaminimizing surfaces. We prove a partial regularity theorem which says that if an $W^{1,2}$ isotropic map from a two-dimensional disk into $\mathbb{R}^{2 n}$ minimizes area relative to its boundary among isotropic competitors and is close enough in $W^{1,2}$ norm to a linear holomorphic isotropic map, then it is smooth in the interior. Furthermore, we prove that the solution to the isotropically constrained Plateau problem exists and has a smooth interior with possibly isolated singularities.
\end{abstract}

\section{Introduction.}

In $[\mathrm{S}-\mathrm{W}]$, Schoen and Wolfson used variational approach to study the existence of special Lagrangian surfaces. They pointed out that if a smooth closed Lagrangian submanifold in a Kähler-Einstein manifold is stationary for the volume functional among all Lagrangian variations, then it is in fact minimal(hence special Lagrangian). Thus they suggested that minimizing volume among all Lagrangian cycles in a homology class will lead to the production of a closed special Lagrangian submanifold if the minimizer is smooth. However, the smoothness of the volume minimizers is not so clear. Schoen and Wolfson studied this regularity problem in twodimensional case. They proved that a Lagrangian map from a 2-dimensional disk to a 4-dimensional Kähler manifold which minimizes area among all Lagrangian maps in the same homology class is in fact a branched immersion away from a finite set of points. As a consequence, they produced branched immersed 2-dimensional Lagrangian cycles with finite number of singularities which minimizes area among its Lagrangian homology class.

In this present paper, using the similar approach of Schoen and Wolfson, we extend their regularity result to higher Co-dimensions. We study the constrained area minimizing problem among the class of isotropic surfaces. In particular we concentrate on the following two-dimensional isotropically constrained Plateau problem in $\mathbb{R}^{2 n}$. 
Let $\omega=\sum_{k=1}^{n} d x^{k} \wedge d y^{k}$ be the standard symplectic form on $\mathbb{R}^{2 n}$. A surface $\Sigma$ is called isotropic if $\left.\omega\right|_{\Sigma}=0$. Let $D_{1}$ be the unit disk in $\mathbb{R}^{2}$. Let $\Gamma$ be a closed piecewise $C^{1}$ Jordan curve in $\mathbb{R}^{2 n}$ which bounds some isotropic disk. It is natural to ask whether $\Gamma$ bounds an isotropic disk which has the least area among all isotropic disks bounded by $\Gamma$. More precisely, define

$$
\begin{aligned}
\mathcal{X}_{\Gamma, I}=\{l \in & W^{1,2}\left(D_{1}, \mathbb{R}^{2 n}\right): l^{*} \omega=0, \\
& \left.\left.l\right|_{\partial D_{1}} \text { is continous and monotone onto } \Gamma\right\}
\end{aligned}
$$

The isotropically constrained Plateau problem then asks two questions:

(1) Does there exist an area minimizer in this class?

(2) What is the regularity of this area minimizer if it exists?

It is fairly easy to get a positive answer to the first question. We shall show in section 2 that the set of weakly isotropic maps is weakly closed. The existence of area minimizer in this class then follows from standard arguments. We will call it a weak solution to the isotropically constrained Plateau problem (Minicozzi in his Ph.D thesis [Mi] gave the similar weak existence of the solution to Legendrianly constrained Plateau problem).

The regularity of this solution turns out to be a much harder problem. We shall first extend Schoen and Wolfson's monotonicity formula for Lagrangian stationary surfaces in $\mathbb{R}^{4}([\mathrm{~S}-\mathrm{W}])$ to higher co-dimensional isotropically stationary surfaces in $\mathbb{R}^{2 n}$. Consequently we deduce a partial regularity results which says that if an $W^{1,2}$ isotropic map from a two-dimensional disk into $\mathbb{R}^{2 n}$ minimizes area relative to its boundary among isotropic competitors and is close enough in $W^{1,2}$ norm to a linear holomorphic isotropic map, then it is smooth interiorly(Theorem 5.9) . By studying the stability of isotropically stationary tangent cones and by using the dimension reduction argument, we obtain the interior regularity theorem which concludes that the solution to the isotropically constrained Plateau problem is smooth away from a finite set(Theorem 7.4).

The organization of this paper is as follows:

First we introduce the weakly isotropic maps and prove the weak existence of the solution to the isotropically constrained Plateau problem(section 2 ). In section 3 we present the monotonicity for isotropically stationary surfaces. In section 4 we prove the excess decay lemma and the consequent $C^{1, \mu}$ partial regularity theorem. The higher regularity issue(from $C^{1, \mu}$ to $C^{\infty}$ ) is of a subtle nature and will be addressed in section 5 . In section 6 we classify all the isotropically stationary tangent cones. Then in the last section we give the interior regularity result. 
I would like very much to thank my advisor Prof. Richard Schoen, whose superb directions and constant encouragements are the main motivation of this paper. I would also like to thank Prof. Leon Simon, Prof. Brian White and Prof. Jon Wolfson for many useful discussions.

\section{Existence of Weak solutions to the Isotropically Constrained Plateau Problem.}

In this section we will introduce the isotropically constrained Plateau problem and prove the weak existence result.

Let $\omega=\sum_{k=1}^{n} d x^{k} \wedge d y^{k}$ be the standard symplectic form in $\mathbb{R}^{2 n}$. A surface $\Sigma^{2} \subset \mathbb{R}^{2 n}$ is called isotropic if $\left.\omega\right|_{\Sigma}=0$.

Definition 2.1. Let $\Sigma^{2} \subset \mathbb{R}^{2 n}$ be an isotropic surface. A deformation $F_{s}$ on $\mathbb{R}^{2 n}$ is called isotropic with respect to $\Sigma$ if $F_{s}(\Sigma)$ are isotropic for all small $s$.

$\Sigma$ is called isotropically stationary if

$$
\left.\frac{d\left(\operatorname{Area}\left(F_{s}\left(\Sigma_{s}\right)\right)\right)}{d s}\right|_{s=0}=0
$$

for any isotropic deformation $F_{s}$ which is compactly supported on $\Sigma$.

We will use $\eta=\sum_{k=1}^{n}\left(x^{k} d y^{k}-y^{k} d x^{k}\right)$ to denote a primitive of $\omega$ in $\mathbb{R}^{2 n}$, use $D_{r}(t)$ to denote the disk in $\mathbb{R}^{2}$ centered at $t$ with radius $r$, and use $C_{r}(t)$ to denote $\partial D_{r}(t)$. In particular, $D_{r}$ will be used to denote $D_{r}(0)$.

Definition 2.2. Let $\Omega \subset \mathbb{R}^{2}$ be a domain. $l \in W^{1,2}\left(\Omega, \mathbb{R}^{2 n}\right)$ is called weakly isotropic if $l^{*} \omega=0$ for a.e. $t \in \Omega$. We will use $W_{I}^{1,2}\left(\Omega, \mathbb{R}^{2 n}\right)$ to denote the set of all weakly isotropic maps.

$l \in W^{1,2}\left(\Omega, \mathbb{R}^{2 n}\right)$ is called weakly conformal if

$$
\left\|l_{*} \frac{\partial}{\partial t^{1}}\right\|=\left\|l_{*} \frac{\partial}{\partial t^{2}}\right\|,\left\langle l_{*} \frac{\partial}{\partial t^{1}}, l_{*} \frac{\partial}{\partial t^{2}}\right\rangle=0, \quad \text { a.e. } t \in \Omega
$$

Lemma 2.3. The set

$$
\Gamma_{I, M}=\left\{l \in W_{I}^{1,2}\left(D_{1}, \mathbb{R}^{2 n}\right): E(l) \leq M\right\}
$$

is weakly closed, where $E(l)=\int_{D_{1}}|\nabla l|^{2} d t$ is the energy of $l$. 
Proof. Let $\left\{l_{i}\right\}_{j=1}^{\infty} \subset \Gamma_{I, M}$. Assume it converges weakly to $l$. Then by the lower-semi-continuity of energy, $E(l) \leq M$. So it remains to show that $l$ is weakly isotropic.

Let $\eta=\sum_{k=1}^{n}\left(x^{k} d y^{k}-y^{k} d x^{k}\right)$ be a primitive of $\omega$ in $\mathbb{R}^{2 n}$. It is easy to see that a map $h$ is weakly isotropic if and only if $\int_{C_{r}(t)} h^{*} \eta=0$ for a.e. $t \in D_{1}$ and $r \in(0,1-|t|)$.

By Rellich lemma, since $E\left(l_{i}\right) \leq M$, we might assume that (by passing to a subsequence) $\left\{l_{i}\right\}_{j=1}^{\infty}$ converges to $l$ strongly in $L^{2}$. Co-area formula then implies that for a.e. $C_{r}(t) \subset D_{1},\left.l_{i}\right|_{C_{r}(t)}$ converges to $\left.l\right|_{C_{r}(t)}$ strongly in $L^{2}$ and weakly in $W^{1,2}$. Hence,

$$
\lim _{i \rightarrow \infty} \int_{C_{r}(t)} l_{i}^{*} \eta=\int_{C_{r}(t)} l^{*} \eta
$$

which shows that $\int_{C_{r}(t)} l^{*} \eta=0$.

The isoperimetric inequality in the setting of isotropic surfaces is very important to construct comparison surfaces. It is proven by Gromov $[\mathrm{Gr}]$ and Allcock $[\mathrm{Al}]$.

Proposition 2.4 (Gromov, Allcock). Given any $W^{1,2}$ map $\phi$ from the unit circle $C$ into $\mathbb{R}^{2 n}$ satisfying $\int_{C} \phi^{*} \sum_{k=1}^{n}\left(x^{k} d y^{k}-y^{k} d x^{k}\right)=0$, there exists a weakly isotropic map $l \in W^{1,2}\left(D_{1}, \mathbb{R}^{2 n}\right)$, such that $l=\phi$ on $C$ and

$$
\operatorname{Area}\left(L\left(D_{1}\right)\right) \leq \text { Length }(l(C))^{2} .
$$

Using the isoperimetric inequality, we are able to prove the Hölder continuity of isotropically area minimizing maps.

Proposition 2.5. If $l \in W^{1,2}\left(D_{1}, \mathbb{R}^{2 n}\right)$ is weakly isotropic, weakly conformal and minimizes area among all isotropic maps with the same boundary value. Then there is a $\mu \in(0,1)$ and an absolute constant $c$ such that $l \in C^{0, \mu}\left(D_{1 / 2}\right)$. Moreover

$$
|l(P)-l(Q)| \leq c A\left(l\left(D_{1}\right)^{1 / 2}|P-Q|^{\mu} \text {, for any } P, Q \in D_{1 / 2} .\right.
$$

Proof. By scaling, we may assume $\operatorname{Area}\left(D_{1}\right)=1$. Given any $p \in D_{1 / 2}$, let $f(r)=E\left(\left.l\right|_{D_{r}(p)}\right)$. The conformality of $l$ implies $f(r)=2 \operatorname{Area}\left(\left.l\right|_{D_{r}(p)}\right)$. Since $l$ is isotropic, we have $\int_{C_{r}(p)} l^{*} \eta=0$. Therefore Proposition 2.4 in additional to the fact that $l$ is isotropically area minimizing, gives that

$$
\operatorname{Area}\left(\left.l\right|_{D_{r}(p)}\right) \leq \text { cLength }\left(\left.l\right|_{C_{r}(p)}\right)^{2} .
$$


Using Co-area formula and Cauchy-Schwartz inequality, it is easy to obtain

$$
\text { Length }\left(\left.\right|_{C_{r}(p)}\right)^{2} \leq 2 \pi r f^{\prime}(r) .
$$

Therefore $f(r) \leq c r f^{\prime}(r)$, where $c>0$ is an absolute constant. Integrating, we get

$$
f(r) \leq\left(f\left(r_{1}\right) r_{1}^{-c}\right) r^{c}
$$

for any $r \leq r_{1}$ such that $D_{r_{1}}(p) \subset D_{1}$. Since $p \in D_{1 / 2}$, we could let $r_{1}=1 / 3$. Note $f(1 / 3) \leq \operatorname{Area}\left(D_{1}\right)=1$. So we have

$$
\int_{D_{r}(p)}|\nabla l|^{2} d t \leq c_{1} r^{c}
$$

where $c_{1}$ and $c$ are absolute positive constant. Morrey's estimate(see [G-T]) implies that $l$ is $C^{0, \mu}$ for some $\mu \in(0,1)$ and

$$
|l(P)-l(Q)| \leq c_{2}|P-Q|^{\mu}
$$

for any $P, Q$ in $D_{1 / 2}$, where $c_{2}$ is an absolute constant.

Definition 2.6. A weakly isotropic map $l: D_{1} \rightarrow \mathbb{R}^{2 n}$ is called exact if there is a $W^{1,2}$ function $\phi$ on $D_{1}$ such that $d \phi=l^{*} \sum_{k=1}^{n}\left(x^{k} d y^{k}-y^{k} d x^{k}\right)$ (we will call $\phi$ a lifting function of $l$ ).

If an isotropic map is exact then we could use the function $\phi$ to lift the map into a contact map in $\mathbb{R}^{2 n+1}$ with the standard contact structure $\alpha=d \phi-\sum_{k=1}^{n}\left(x^{k} d y^{k}-y^{k} d x^{k}\right)$. More precisely, for a weakly exact isotropic map $l: D_{1} \rightarrow \mathbb{R}^{2 n}$, we can define a map $\tilde{l}: D_{1} \rightarrow \mathbb{R}^{2 n+1}$ by

$$
\tilde{l}(t)=(l(t), \phi(t)) \in \mathbb{R}^{2 n+1} .
$$

Then clearly $\tilde{l}^{*} \alpha=0$.

Proposition 2.7. If $l \in W^{1,2}\left(D_{1}, \mathbb{R}^{2 n}\right)$ is weakly isotropic, weakly conformal and minimizes area among all isotropic maps with the same boundary value. Then $l$ is locally exact.

Proof. Proposition 2.5 implies $l$ is continuous. Local integrating gives the existence of the lifting function $\phi$. 
Now we consider the isotropically constrained Plateau problem. Let $\Gamma$ be a piecewise $C^{1}$ closed Jordan curve such that $\int_{\Gamma} \eta=0$. Define

$$
\begin{aligned}
\mathcal{X}_{\Gamma, I}=\{l \in & W_{I}^{1,2}\left(D_{1}, \mathbb{R}^{2 n}\right): \\
& \left.\left.l\right|_{\partial D_{1}} \text { is continous and is a monotone map onto } \Gamma\right\}
\end{aligned}
$$

By Proposition 2.4, $\Gamma$ bounds some isotropic disk, so $\mathcal{X}_{\Gamma, I}$ is not empty.

Let $A_{\Gamma, I}=\inf _{l \in \mathcal{X}_{\Gamma, I}}$ Area $(l)$ and let $E_{\Gamma, I}=\inf _{l \in \mathcal{X}_{\Gamma, I}} E(l)$. Classical result in Plateau problem gives:

Lemma 2.8. $A_{\Gamma, I}=\frac{1}{2} E_{\Gamma, I}$. Moreover if $E\left(l_{0}\right)=E_{\Gamma, I}$, then $A\left(l_{0}\right)=A_{\Gamma, I}$.

The above lemma enables us to minimize energy instead of area. To quotient out the conformal group of the disk, we consider a subclass. Let $p_{1}, p_{2}$ and $p_{3}$ be distinct points in $\partial D_{1}$ and $q_{1}, q_{2}$ and $q_{3}$ be distinct points on $\Gamma$. Define

$$
\mathcal{X}_{\Gamma, I}^{\prime}=\left\{l \in \mathcal{X}_{\Gamma, I}:\left.l\right|_{\partial D_{1}}\left(p_{i}\right)=q_{i}, i=1,2,3\right\} .
$$

We now have the following existence theorem of the isotropically constrained Plateau problem.

Theorem 2.9. There is a weakly conformal, weakly isotropic map $l_{0} \in \mathcal{X}_{\Gamma, I}$ such that $A\left(l_{0}\right)=A_{\Gamma, I}$. Moreover $l_{0}$ is Hölder continuous in the interior of $D_{1}$.

Proof. Since energy is conformally invariant, we see that

$$
\inf _{l \in \mathcal{X}_{\Gamma, I}^{\prime}} E(l)=E_{\Gamma, I}
$$

It suffices to show that there is an energy minimizer in $\mathcal{X}_{\Gamma, I}^{\prime}$. Let $\left\{l_{i}\right\}_{i=1}^{\infty} \subset$ $\mathcal{X}_{\Gamma, I}^{\prime}$ such that $\lim _{i \rightarrow \infty} E\left(l_{i}\right)=E_{\Gamma, I}$. Assume $\left\{l_{i}\right\}_{i=1}^{\infty}$ converges weakly to $l_{0}$ in $W^{1,2}$ sense and strongly in $L^{2}$ sense. The lower-semi-continuity of energy gives $E\left(l_{0}\right) \leq E_{\Gamma, I}$. It suffices now to show that $l_{0}$ is in the class of $\mathcal{X}_{\Gamma, I}^{\prime}$. Lemma 2.3 shows that $l_{0}$ is weakly isotropic. Classical Courant-Lebesgue lemma(see $[\mathrm{La}]$ ) gives that the class $\left\{\left.l\right|_{\partial D_{1}}: l \in \mathcal{X}_{\Gamma, I}^{\prime}\right\}$ is equicontinuous. Therefore by Arzela-Ascoli lemma we might assume(by passing to a subsequence) $\left\{\left.l_{i}\right|_{\partial D_{1}}\right\}_{i=1}^{\infty}$ converges in $C^{0}$ sense to a continuous function $\varphi: \partial D_{1} \rightarrow \mathbb{R}^{2 n}$. Clearly $\varphi$ is monotone onto $\Gamma$ and $\varphi\left(p_{i}\right)=q_{i}$ for $i=1,2,3$. We know that the trace map is continuous from 
$W^{1,2}\left(D_{1}, \mathbb{R}^{2 n}\right)$ to $W^{1 / 2,2}\left(\partial D_{1}, \mathbb{R}^{2 n}\right)$, and $W^{1 / 2,2}\left(\partial D_{1}, \mathbb{R}^{2 n}\right)$ is compactly embedded in $L^{2}\left(\partial D_{1}, \mathbb{R}^{2 n}\right)$. Therefore the weak convergence of $\left\{l_{i}\right\}_{i=1}^{\infty}$ to $l_{0}$ in $W^{1,2}\left(D_{1}, \mathbb{R}^{2 n}\right)$ implies the strong convergence of $\left\{\left.l_{i}\right|_{\partial D_{1}}\right\}_{i=1}^{\infty}$ to $\left.l_{0}\right|_{\partial D_{1}}$ in $L^{2}\left(\partial D_{1}, \mathbb{R}^{2 n}\right)$. Hence we $\left.l_{0}\right|_{\partial D_{1}}=\varphi$. So $\left.l_{0}\right|_{\partial D_{1}}$ is continuous, monotone onto $\Gamma$ and $\left.l_{0}\right|_{\partial D_{1}}\left(p_{i}\right)=q_{i}$. Hence $l_{0} \in \mathcal{X}_{\Gamma, I}^{\prime}$ and $E(l)=E_{\Gamma, I}$. By Lemma $2.8, l_{0}$ is the area minimizer. The Hölder continuity follows from Proposition 2.5.

\section{Extension of Schoen-Wolfson's Monotonicity to Higher Co-dimensions.}

Monotonicity for two-dimensional Lagrangian stationary surfaces was obtained by Schoen and Wolfson in $[\mathrm{S}-\mathrm{W}]$. With a slight modification we can extend their result to higher co-dimensions.

Instead of deriving the monotonicity directly in the Lagrangian setting, Schoen and Wolfson consider the lifting of a Lagrangian surfaces into a contact space and derive the monotonicity upstairs. Let $\left(x^{1}, \ldots, x^{n}, y^{1}, \ldots, y^{n}, \varphi\right)$ be the coordinate on $\mathbb{R}^{2 n+1}$. And let $\alpha=d \varphi-\eta$ be the standard contact form on $\mathbb{R}^{2 n+1}$, where $\eta=\sum_{k=1}^{n}\left(x^{k} d y^{k}-y^{k} d x^{k}\right)$. A submanifold $N^{k}$ is called contact if $\left.\alpha\right|_{N}=0$. A vector field $X$ in $\mathbb{R}^{2 n+1}$ is called a contact vector field if its flow preserves the contact structure, i.e. $L_{X} \alpha=f \alpha$ for some function $f$. Just like in the symplectic case, any ambient function in $\mathbb{R}^{2 n+1}$ generates a contact vector field.

Lemma 3.1 (Schoen-Wolfson $[\mathbf{S}-\mathbf{W}])$ ). Let $h: \mathbb{R}^{2 n+1} \rightarrow \mathbb{R}$ be any smooth function. Define a vector field

$$
\begin{aligned}
X_{h}= & h_{x} \frac{\partial}{\partial y}-h_{y} \frac{\partial}{\partial x}-h_{\varphi}\left(x \frac{\partial}{\partial x}+y \frac{\partial}{\partial y}\right) \\
& +\left(-2 h+x h_{x}+y h_{y}\right) \frac{\partial}{\partial \varphi} .
\end{aligned}
$$

Then $X_{h}$ is a contact vector field(and will be called the Hamiltonian vector field for $h$ ).

Define $\Pi: \mathbb{R}^{2 n+1} \rightarrow \mathbb{R}^{2 n}$ to be the projection along $\varphi$ direction. For vectors $Z_{1}, Z_{2}$ in $\mathbb{R}^{2 n+1}$, define

$$
\left\langle Z_{1}, Z_{2}\right\rangle_{d}=\left\langle\Pi_{*} Z_{1}, \Pi_{*} Z_{2}\right\rangle
$$


where $\langle$,$\rangle is the standard Euclidean metric on \mathbb{R}^{2 n}$. Note that $\langle,\rangle_{d}$ is not a Riemannian metric on $\mathbb{R}^{2 n+1}$ since it degenerates. But it is not difficult to see that the restriction of $\langle,\rangle_{d}$ to any contact linear subspace is non-degenerate.

Let $l: D_{1} \rightarrow \mathbb{R}^{2 n}$ be an isotropic map. Assume $l$ is exact and isotropically stationary(in the sense that the first variation of area is zero for compactly supported deformations of $l$ through isotropic maps). Then as described in section 2 , the exactness of $l$ gives a lifting map $\tilde{l}: D_{1} \rightarrow \mathbb{R}^{2 n+1}$ via $\tilde{l}=(l, \varphi)$. Now let $Z$ be any contact vector field in $\mathbb{R}^{2 n+1}$ such that $Z$ vanishes along $\tilde{l}\left(\partial D_{1}\right)$ and let $F_{t}$ be the flow generated by $Z$. Define $\tilde{l}_{t}:=F_{t} \circ \tilde{l}$ and define an isotropic deformation $l_{t}$ of $l$ by

$$
l_{t}:=\Pi \circ \tilde{l}_{t}
$$

Then first variation of area gives

Proposition 3.2 (Schoen-Wolfson $[\mathbf{S}-\mathbf{W}]$ ). Let $l: D_{1} \rightarrow \mathbb{R}^{2 n}$ be an isotropic map which is exact and isotropically stationary. Let $\tilde{l} \rightarrow \mathbb{R}^{2 n+1}$ be the lifting of $l$. If $Z$ is a contact vector field in $\mathbb{R}^{2 n+1}$ such that $Z$ vanishes along $\tilde{l}\left(\partial D_{1}\right)$, then

$$
\int_{\Sigma} \operatorname{div}_{\Sigma} Z d \mu_{\Sigma}=0
$$

Here $\operatorname{div}_{\Sigma} Z:=\sum_{k=1}^{2}\left\langle\nabla_{e_{k}}^{0} Z, e_{k}\right\rangle_{d}$, where $\nabla^{0}$ denotes the Euclidean connection on $\mathbb{R}^{2 n+1}$ and $\left\{e_{i}\right\}_{i=1}^{2}$ is a set of orthonormal tangent vector of $\Sigma:=\tilde{l}\left(D_{1}\right)$ with respect to the metric $\langle,\rangle_{d}$.

The idea to get the monotonicity is to find a proper Hamiltonian function $h$ and apply Proposition 3.2 to the contact vector field $X_{h}$.

Let $s=2^{-1}\left(x^{2}+y^{2}\right)$ and

$$
r=\sqrt{2}\left(s^{2}+\varphi^{2}\right)^{1 / 4}
$$

$r$ plays the same role as the distance function and will be called the modified distance function in $\mathbb{R}^{2 n+1}$. Define

$$
\tilde{B}_{\sigma}(0)=\left\{P \in \mathbb{R}^{2 n+1}: r(P)<\sigma\right\} .
$$

Also define a change of variables,

$$
t=\log \left(\sqrt{s^{2}+\varphi^{2}}\right), \quad \theta=\arctan \frac{\varphi}{s} .
$$


Since $l\left(D_{1}\right) \subset \mathbb{R}^{2 n}$ is isotropic, we have the orthogonal decomposition of the tangent space of $\mathbb{R}^{2 n}$ :

$$
T \mathbb{R}^{2 n}=\operatorname{Tl}\left(D_{1}\right) \oplus \operatorname{JTl}\left(D_{1}\right) \oplus Q
$$

where $J$ is the standard complex structure of $\mathbb{R}^{2 n}$. Let $\pi: T \mathbb{R}^{2 n} \rightarrow Q$ be the orthogonal projection.

Similiar computation as in Schoen-Wolfson's paper [S-W] yields the following equation(the proof of which will be omitted here)

Proposition 3.3. Let $h(t, \theta): \mathbb{R}^{2 n+1} \rightarrow \mathbb{R}$ be a smooth function. Then

$$
\begin{aligned}
& \operatorname{div}_{\Sigma} X_{h(t, \theta)} \\
= & -2 h_{t} e^{-t} \sin \theta-2 h_{\theta t} e^{-t} \cos \theta \\
& +\left(h_{\theta t}-h_{\theta}\right)\left(2\left\|\nabla^{\Sigma} \theta\right\|_{d}^{2}+e^{-2 t}\|\pi(\vec{P})\|^{2}\right) \\
& +\left\{-2 h_{t}+h_{t t}-h_{\theta \theta}\right\}\left\langle\nabla^{\Sigma} \theta, \nabla^{\Sigma} t\right\rangle_{d},
\end{aligned}
$$

where $\vec{P}$ is the position vector of $l$ in $\mathbb{R}^{2 n}$.

Once Proposition 3.3 is established, we could use the exactly same argument in Schoen-Wolfson's paper to derive the monotonicity. For the convenience of future use, we include the argument here.

Fix $a>0$, let $\rho_{a}(t)$ to be a smooth function of $t$ such that

$$
\rho_{a}(t)= \begin{cases}1-2 a^{-2} e^{t} & \text { if } t \leq \log \left(a^{2} / 2\right)-1, \\ 0 & \text { if } t \geq \log \left(a^{2} / 2\right)\end{cases}
$$

Let

$$
\begin{gathered}
\Gamma_{1}=\left\{(t, \theta): \theta \in\left(-\frac{\pi}{2}, \frac{\pi}{2}\right), \theta \leq-t-\log \left(\frac{a^{2}}{2}\right)-1, \theta \geq t-\log \left(\frac{a^{2}}{2}\right)-1\right\}, \\
\Gamma_{2}=\left\{(t, \theta): \theta \in\left(-\frac{\pi}{2}, \frac{\pi}{2}\right), \theta \geq-t-\log \left(\frac{a^{2}}{2}\right), \theta \leq t-\log \left(\frac{a^{2}}{2}\right)\right\} \\
\Gamma_{3}=\left\{(t, \theta): \theta \in\left(-\frac{\pi}{2}, \frac{\pi}{2}\right)\right\} \backslash\left(\Gamma_{1} \bigcup \Gamma_{2}\right) .
\end{gathered}
$$

Consider the following wave equation:

$$
\left\{\begin{array}{l}
h_{t t}-h_{\theta \theta}-2 h_{t}=0 \\
h(t, 0)=0 \\
h_{\theta}(t, 0)=\rho_{a}(t)
\end{array}\right.
$$


Clearly we see that the solution of equation(9) is given by

$$
h^{(a)}= \begin{cases}\theta-2 a^{-2} e^{-t} \sin \theta & \text { if }(t, \theta) \in \Gamma_{1}, \\ * * & \text { if }(t, \theta) \in \Gamma_{3}, \\ 0 & \text { if }(t, \theta) \in \Gamma_{2},\end{cases}
$$

where $* *$ is some smooth function. Define

$$
\begin{aligned}
G^{(a)} & =h_{\theta}^{(a)}-h_{\theta t}^{(a)}, \\
F^{(a)} & =\frac{1}{2} a^{2} e^{-t}\left(h_{\theta t}^{(a)} \cos \theta+h_{t}^{(a)} \sin \theta\right) .
\end{aligned}
$$

Then $G^{(a)}=1$ and $F^{(a)}=1$ in $\Gamma_{1}$. By scaling,

$$
\begin{aligned}
& F^{(a)}(t, \theta)=F^{(1)}\left(t-\log \left(a^{2} / 2\right), \theta\right), \\
& G^{(a)}(t, \theta)=G^{(1)}\left(t-\log \left(a^{2} / 2\right), \theta\right) .
\end{aligned}
$$

Schoen and Wolfson $[\mathrm{S}-\mathrm{W}]$ proved the following proposition concerning $F^{(a)}$ and $G^{(a)}$ :

Proposition 3.4 (Schoen-Wolfson). The function $F^{(a)}$ is non-negative. And $G$ satisfies $0 \leq G^{(a)} \leq 1$ for $\theta \in\left(-\frac{\pi}{2}, \frac{\pi}{2}\right)$. Furthermore, there is a fixed constant $\theta_{0}$ such that $G^{(a)}-G^{(b)} \geq 0$ for $0 \leq b \leq \theta_{0} a$.

Now by Proposition 3.3, we have

$$
\operatorname{div}_{\Sigma} X_{h^{(a)}}=4 a^{-2} F^{(a)}-\left(2\left\|\nabla^{\Sigma} \theta\right\|_{d}^{2}+e^{-2 t}\|\pi(\vec{P})\|^{2}\right) G^{(a)} .
$$

The support of $h^{(a)}$ is contained in

$$
\left\{(t, \theta): \theta \in\left(-\frac{\pi}{2}, \frac{\pi}{2}\right), t \leq \log \left(a^{2} / 2\right)+\pi / 2\right\}
$$

which is in fact $\tilde{B}_{\sqrt{2} e^{\pi / 4} a}(0)$ in $\mathbb{R}^{2 n+1}$. Assume $0<b<a$, then $h^{(a)}-$ $h^{(b)}$ is a smooth function compactly supported in $\tilde{B}_{\sqrt{2} e^{\pi / 4} a}(0)$. Therefore Proposition 3.2 and (11) imply,

$$
\begin{aligned}
& a^{-2} \int_{\Sigma} F^{(a)} d \mu_{\Sigma}-b^{-2} \int_{\Sigma} F^{(b)} d \mu_{\Sigma} \\
= & 2^{-1} \int_{\Sigma}\left(G^{(a)}-G^{(b)}\right)\left(\left\|\nabla^{\Sigma} \theta\right\|_{d}^{2}+2^{-1} e^{-2 t}\|\pi(\vec{P})\|^{2}\right) d \mu_{\Sigma} .
\end{aligned}
$$

Combine Proposition 3.4 and (12), we have 
Theorem 3.5 (Monotonicity). Let $l: D_{1} \rightarrow \mathbb{R}^{2 n}$ be a weakly isotropic, weakly conformal, exact, and isotropically stationary map with a contact lifting $\tilde{l}: D_{1} \rightarrow \mathbb{R}^{2 n}$. Let $\Sigma=\tilde{l}\left(D_{1}\right)$. Assume $\tilde{l}(0)=0$ and $\tilde{l}\left(\partial D_{1}\right)$ is outside $\tilde{B}_{r}(0)$ (defined in (7)). Then there are constants $c_{1}$ and $c_{2}$ which only depend on $\operatorname{Area}(\Sigma)$ and $r$ such that

$$
c_{1} \leq \sigma^{-2} \operatorname{Area}\left(\Sigma \cap \tilde{B}_{\sigma}(0)\right) \leq c_{2},
$$

for any $\sigma \in(0, r)$.

\section{4. $C^{1, \mu} \varepsilon$-Regularity for mapping problem.}

Through out this section we will assume $l \in W^{1,2}\left(D_{2}, \mathbb{R}^{2 n}\right)$ is weakly isotropic, weakly conformal and area-minimizing among all isotropic maps with the same boundary value. By Proposition $2.7, l$ is locally exact. Therefore monotonicity applies. Also proposition 2.5 tells us that $l$ is actually $C^{0, \mu}$.

First quote a reversed Poincaré type inequality which is proven by Schoen and Wolfson $[\mathrm{S}-\mathrm{W}]$.

Proposition 4.1 (Schoen-Wolfson). Assume that $A\left(l\left(D_{2}\right)\right) \leq c_{1}$, and $l_{0}$ is a linear holomorphic map into a isotropic plane in $\mathbb{R}^{2 n}$. For any $\varepsilon>0$, there exists an absolute constant $c$ and a constant $\delta=\delta(\varepsilon)$ such that if

$$
\int_{D_{2}}\left\{\left|\nabla\left(l-l_{0}\right)\right|^{2}+\left|l-l_{0}\right|^{2}\right\} d t \leq \delta
$$

then

$$
\int_{D_{1}}\left|\nabla\left(l-l_{0}\right)\right|^{2} d t \leq \varepsilon \int_{D_{2}}\left|\nabla\left(l-l_{0}\right)\right|^{2} d t+c \int_{D_{2}}\left|l-l_{0}\right|^{2} d t .
$$

Now we define the excess type quantity

$$
E\left(l, l_{0}, r\right)=r^{-2} \int_{D_{r}}\left|\nabla\left(l-l_{0}\right)\right|^{2} d t,
$$

where $l_{0}$ is a linear holomorphic map into an isotropic plane in $\mathbb{R}^{2 n}$.

Lemma 4.2 (Excess Decay Lemma). Assume $A\left(l\left(D_{1}\right)\right) \leq c_{1}$. There exist constants $\varepsilon>0$ and $\bar{\theta} \in(0,1)$ depending only on $c_{1}$ and an absolute constant $c$ such that if

$$
E\left(l, l_{0}, 1\right)+\int_{D_{1}}\left|l-l_{0}\right|^{2} d t \leq \varepsilon
$$


then there exists a linear holomorphic map $l_{1}$ into an isotropic plane such that

$$
\begin{gathered}
E\left(l, l_{1}, \bar{\theta}\right) \leq \frac{1}{2} E\left(l, l_{0}, 1\right), \\
\bar{\theta}^{-4} \int_{D_{\bar{\theta}}}\left|l-l_{1}\right|^{2} d t \leq c E\left(l, l_{0}, 1\right) .
\end{gathered}
$$

Proof. Without loss of generality, assume $l_{0}\left(t^{1}, t^{2}\right)=\left(t^{1}, t^{2}, 0,0, \ldots, 0\right)$ is the identity map of $D_{1}$ into the $x^{1} x^{2}$-plane. Assume the lemma is not true, then we have a sequence of maps $l^{(j)}=\left(x^{(j) 1}, \ldots, x^{(j) n}, y^{(j) 1}, \ldots, y^{(j) n}\right)$ such that

$$
E\left(l^{(j)}, l_{0}, 1\right)+\int_{D_{1}}\left|l^{(j)}-l_{0}\right|^{2} d t=\varepsilon_{j},
$$

where $\lim _{j \rightarrow \infty} \varepsilon_{j}=0$, but none of them satisfies (15). Let $E_{j}=E\left(l^{(j)}, l_{0}, 1\right)$. Define

$$
\begin{gathered}
u^{(j) 1}=\frac{1}{\sqrt{E_{j}}}\left(x^{(j) 1}-t^{1}\right)-\alpha^{(j) 1}, \\
u^{(j) 2}=\frac{1}{\sqrt{E_{j}}}\left(x^{(j) 2}-t^{2}\right)-\alpha^{(j) 2}, \\
w^{(j) k}=\frac{1}{\sqrt{E_{j}}} x^{(j) k}-\gamma^{(j) k}, k=3, \ldots, n, \\
v^{(j) 1}=\frac{1}{\sqrt{E_{j}}} y^{(j) 1}-\beta^{(j) 1}, \\
v^{(j) 2}=\frac{1}{\sqrt{E_{j}}} y^{(j) 2}-\beta^{(j) 2}, \\
q^{(j) k}=\frac{1}{\sqrt{E_{j}}} y^{(j) k}-\mu^{(j) k}, k=3, \ldots, n,
\end{gathered}
$$

where $\alpha, \beta, \gamma$, and $\mu$ are constants to make $\int_{D_{1}} u^{(j)}=0, \int_{D_{1}} v^{(j)}=0$, $\int_{D_{1}} w^{(j)}=0$, and $\int_{D_{1}} q^{(j)}=0$. Then clearly

$$
\int_{D_{1}}\left|\nabla u^{(j)}\right|^{2} d t+\int_{D_{1}}\left|\nabla v^{(j)}\right|^{2} d t+\int_{D_{1}}\left|\nabla w^{(j)}\right|^{2} d t+\int_{D_{1}}\left|\nabla q^{(j)}\right|^{2} d t=1 .
$$

Standard Poincaré inequality gives that $u^{(j)}, v^{(j)}, w^{(j)}$ and $q^{(j)}$ are bounded in $W^{1,2}$, so by Alaoglu Theorem and Rellich's Theorem we can assume(by passing to a subsequence) that they converge to $u, v, w, q$ respectively 
weakly in $W^{1,2}$ and strongly in $L^{2}$. The lower-semi-continuity of energy gives

$$
\int_{D_{1}}|\nabla u|^{2} d t+\int_{D_{1}}|\nabla v|^{2} d t+\int_{D_{1}}|\nabla w|^{2} d t+\int_{D_{1}}|\nabla q|^{2} d t \leq 1 .
$$

On the other hand, from (16), we know that $x^{(j) 1}\left(t^{1}, t^{2}\right)$ converges strongly to $t^{1}$ in $L^{2}, x^{(j) 2}\left(t^{1}, t^{2}\right)$ converges strongly to $t^{2}$ in $L^{2}$ and $x^{(j) k}\left(t^{1}, t^{2}\right), y^{(j) k}\left(t^{1}, t^{2}\right)$ converge strongly to 0 in $L^{2}$ sense.

Claim 1. $u$ is a holomorphic from $D_{1}$ to $x^{1} x^{2}$-plane.

Proof. Let $t=t^{1}+\sqrt{-1} t^{2}, x=x^{1}+\sqrt{-1} x^{2}, y=y^{1}+\sqrt{-1} y^{2}$, and $z^{k}=$ $x^{k}+\sqrt{-1} y^{k}$ for $k=3, \ldots, n$. Then the conformality of $l^{(j)}$ gives us

$$
\frac{\partial x^{(j)}}{\partial t} \frac{\partial \bar{x}^{(j)}}{\partial t}+\frac{\partial y^{(j)}}{\partial t} \frac{\partial \bar{y}^{(j)}}{\partial t}+\sum_{k=3}^{n} \frac{\partial z^{(j) k}}{\partial t} \frac{\partial \bar{z}^{(j)} k}{\partial t}=0
$$

Cauchy -Schwartz inequality implies

$$
\int_{D_{1}}\left|\frac{\partial x^{(j)}}{\partial t} \frac{\partial \bar{x}^{(j)}}{\partial t}\right| d t \leq \sum_{\alpha=1}^{2} \int_{D_{1}}\left|\nabla y^{(j) \alpha}\right|^{2} d t+\sum_{k=3}^{n} \int_{D_{1}}\left(\left|\nabla y^{(j) k}\right|^{2}+\left|\nabla x^{(j) k}\right|^{2}\right) d t
$$

Using the fact that $\frac{\partial \bar{x}^{(j)}}{\partial t}=\frac{\partial x^{(j)}}{\partial t} \frac{\partial \bar{x}^{(j)}}{\partial t}-\frac{\partial\left(x^{(j)}-t\right)}{\partial t} \frac{\partial\left(\bar{x}^{(j)}-\bar{t}\right)}{\partial t}$, we get from (20) that

$$
\int_{D_{1}}\left|\frac{\partial \bar{x}^{(j)}}{\partial t}\right| d t \leq \int_{D_{1}}\left|\nabla\left(l-l_{0}\right)\right|^{2} d t=E_{j} .
$$

Thus

$$
\int_{D_{1}}\left|\frac{\partial \bar{u}^{(j)}}{\partial t}\right| d t \leq \sqrt{E_{j}}
$$

Let $j$ go to infinity, we see that $u$ is holomorphic.

Claim 2. There is a biharmonic function $f: D_{1} \rightarrow \mathbb{R}$, such that $\left(v^{1}, v^{2}\right)=$ $\nabla f$. And $w^{k}, q^{k}$ are harmonic functions on $D_{1}$ for $k=3, \ldots, n$.

Proof. Let $\eta, \tilde{x}^{k}, \tilde{y}^{k}, k=3,4, \ldots, n$ be smooth functions of $x^{1}, x^{2}$ with compact support in $\tilde{D}_{1}=\left\{\left(x^{1}, x^{2}\right):\left(x^{1}\right)^{2}+\left(x^{2}\right)^{2} \leq 1\right\}$. Define $\tilde{y}^{1}, \tilde{y}^{2}$ to be functions of $x^{1}, x^{2}, x^{k}, \ldots, y^{k}, k=3, \ldots, n$ by

$$
\tilde{y}^{\alpha}=\sum_{k=3}^{n}\left(x^{k} \frac{\partial \tilde{y}^{k}}{\partial x^{\alpha}}-y^{k} \frac{\partial \tilde{x}^{k}}{\partial x^{\alpha}}\right)+\frac{\partial \eta}{\partial x^{\alpha}}, \alpha=1,2 .
$$


Now let $X$ be a vector field in the cylinder $\tilde{D}_{1} \times \mathbb{R}^{2 n-2}$ defined by

$$
X:=\sum_{k=3}^{n}\left(\tilde{x}^{k} \frac{\partial}{\partial x^{k}}+\tilde{y}^{k} \frac{\partial}{\partial y^{k}}\right)+\sum_{\alpha=1}^{2} \tilde{y}^{\alpha} \frac{\partial}{\partial y^{\alpha}} .
$$

It is easy to check that $d(X\rfloor \omega)=0$. Therefore $X$ is a symplectic vector field, i.e. its flow $F_{s}$ preserves the symplectic form $\omega$ (see [M-S]).

Since $l^{(j)}$ is isotropically area minimizing, by Proposition 2.5 , it is $C^{0, \mu}$ and has uniform $C^{0, \mu}$ bound. Hence we can assume $l^{(j)}-l_{0}$ is pointwise small since its $L^{2}$ norm is small. Therefore, $F_{s} \circ l^{(j)}\left(t^{1}, t^{2}\right)$ has compact support in $D_{1}$ for $j$ sufficiently large. Since $l^{(j)}$ minimizes energy, we get $\left.\frac{d}{d s}\right|_{s=0} \int_{D_{1}}\left|\nabla F_{s} \circ l^{(j)}\right|^{2} d t=0$. Thus we have

$$
\begin{aligned}
& \sum_{k=3}^{n} \int_{D_{1}} \nabla x^{(j) k} \cdot \nabla \tilde{x}^{k}\left(x^{(j) 1}, x^{(j) 2}\right) d t \\
+ & \sum_{k=3}^{n} \int_{D_{1}} \nabla y^{(j) k} \cdot \nabla \tilde{y}^{k}\left(x^{(j) 1}, x^{(j) 2}\right) d t \\
+ & \sum_{\alpha=1}^{2} \int_{D_{1}} \nabla y^{(j) \alpha} \cdot \nabla \tilde{y}^{\alpha}\left(x^{(j) 1}, x^{(j) 2}, \ldots, x^{(j) n}, y^{(j) 3}, \ldots, y^{(j) n}\right) d t, \\
= & 0,
\end{aligned}
$$

where $\nabla$ is the gradient of $t$ in $D_{1}$. Using the chain rule, for example

$$
\nabla \tilde{x}^{k}\left(x^{(j) 1}(t), x^{(j) 2}(t)\right)=\sum_{\alpha=1}^{2} \frac{\partial \tilde{x}^{k}}{\partial x^{\alpha}}\left(x^{(j) 1}(t), x^{(j) 2}(t)\right) \nabla x^{(j) \alpha}
$$


together with (21) and (23), we get

$$
\begin{aligned}
& \sum_{k=3}^{n} \int_{D_{1}} \sum_{\alpha=1}^{2} \frac{\partial \tilde{x}^{k}}{\partial x^{\alpha}}\left(x^{(j) 1}(t), x^{(j) 2}(t)\right) \nabla x^{(j) \alpha} \cdot \nabla x^{(j) k} d t \\
+ & \sum_{k=3}^{n} \int_{D_{1}} \sum_{\alpha=1}^{2} \frac{\partial \tilde{y}^{k}}{\partial x^{\alpha}}\left(x^{(j) 1}(t), x^{(j) 2}(t)\right) \nabla x^{(j) \alpha} \cdot \nabla y^{(j) k} d t \\
+ & \sum_{\alpha=1}^{2} \sum_{k=3}^{n} \int_{D_{1}}\left\{\nabla y ^ { ( j ) \alpha } \cdot \nabla \left[x^{(j) k}(t) \frac{\partial \tilde{y}^{k}}{\partial x^{\alpha}}\left(x^{(j) 1}(t), x^{(j) 2}(t)\right)\right.\right. \\
& \left.\left.-y^{(j) k}(t) \frac{\partial \tilde{x}^{k}}{\partial x^{\alpha}}\left(x^{(j) 1}(t), x^{(j) 2}(t)\right)\right]\right\} d t \\
+ & \sum_{\alpha=1}^{2} \int_{D_{1}} \nabla y^{(j) \alpha} \cdot \nabla \frac{\partial \eta}{\partial x^{\alpha}}\left(x^{(j) 1}(t), x^{(j) 2}(t)\right) d t \\
= & 0 .
\end{aligned}
$$

Now note that $\nabla x^{(j) \alpha}=\nabla\left(x^{(j) \alpha}-t^{\alpha}\right)+\nabla t^{\alpha}$. Therefore

$$
\begin{aligned}
& \quad \mid \sum_{k=3}^{n} \sum_{\gamma=1}^{2}\left(\int_{D_{1}} \frac{\partial x^{(j) k}}{\partial t^{\gamma}} \frac{\partial \tilde{x}^{k}}{\partial x^{\gamma}} d t+\int_{D_{1}} \frac{\partial y^{(j) k}}{\partial t^{\gamma}} \frac{\partial \tilde{y}^{k}}{\partial x^{\gamma}} d t\right) \\
& +\sum_{\alpha, \gamma=1}^{2} \int_{D_{1}} \frac{\partial y^{(j) \alpha}}{\partial t^{\gamma}} \frac{\partial^{2} \eta}{\partial x^{\alpha} \partial x^{\gamma}} d t \mid \\
& \leq \sum_{k=3}^{n} \sum_{\alpha=1}^{2}\left(\tilde{c} \int_{D_{1}}\left\|\nabla\left(x^{(j) \alpha}-t^{\alpha}\right)\right\| \cdot\left\|\nabla x^{(j) k}\right\| d t\right. \\
& +\tilde{c} \int_{D_{1}}\left\|\nabla\left(x^{(j) \alpha}-t^{\alpha}\right)\right\| \cdot\left\|\nabla y^{(j) k}\right\| d t \\
& +\tilde{c} \int_{D_{1}}\left\|\nabla y^{(j) \alpha}\right\| \cdot\left\|\nabla x^{(j) k}\right\| d t+\tilde{c} \int_{D_{1}}\left|x^{(j) k}\right| \cdot\left\|\nabla y^{(j) \alpha}\right\| d t \\
& +\tilde{c} \int_{D_{1}}\left\|\nabla y^{(j) \alpha}\right\| \cdot\left\|\nabla y^{(j) k}\right\| d t+\tilde{c} \int_{D_{1}}^{\left.\left|y^{(j) k}\right| \cdot\left\|\nabla y^{(j) \alpha}\right\| d t\right)}
\end{aligned}
$$

where $\tilde{c}$ depends on the $C^{2}$ norm of $\tilde{x}^{k}, \tilde{y}^{k}$ and $\eta$. All $\tilde{x}^{k}, \tilde{y}^{k} \eta$ and their derivatives above are evaluated at $\left(x^{(j) 1}\left(t^{1}, t^{2}\right), x^{(j) 2}\left(t^{1}, t^{2}\right)\right)$. 
Thus Cauchy-Schwartz inequality implies

$$
\begin{aligned}
& \quad \mid \sum_{k=3}^{n} \sum_{\gamma=1}^{2}\left(\int_{D_{1}} \frac{\partial x^{(j) k}}{\partial t^{\gamma}} \frac{\partial \tilde{x}^{k}}{\partial x^{\gamma}} d t+\int_{D_{1}} \frac{\partial y^{(j) k}}{\partial t^{\gamma}} \frac{\partial \tilde{y}^{k}}{\partial x^{\gamma}} d t\right) \\
& \quad+\sum_{\alpha, \gamma=1}^{2} \int_{D_{1}} \frac{\partial y^{(j) \alpha}}{\partial t^{\gamma}} \frac{\partial^{2} \eta}{\partial x^{\alpha} \partial x^{\gamma}} d t \mid \\
& \leq \quad \tilde{c} E\left(l^{(j)}, l_{0}, 1\right) \\
& \quad+\sum_{k=3}^{n} \sum_{\alpha=1}^{2}\left(\tilde{c} \int_{D_{1}}\left|x^{(j) k}\right| \cdot|| \nabla y^{(j) \alpha}|| d t+\tilde{c} \int_{D_{1}}\left|y^{(j) k}\right| \cdot|| \nabla y^{(j) \alpha} \| d t\right) .
\end{aligned}
$$

By Cauchy-Schwartz,

$$
\int_{D_{1}}\left|y^{(j) k}\right| \cdot\left\|\nabla y^{(j) \alpha}\right\| d t \leq\left\|y^{(j) k}\right\|_{L^{2}\left(D_{1}\right)} E^{1 / 2}\left(l^{(j)}, l_{0}, 1\right) .
$$

Therefore combine (17), (24) and (25) we get

$$
\begin{aligned}
& \mid \sum_{k=3}^{n} \sum_{\gamma=1}^{2}\left(\int_{D_{1}} \frac{\partial w^{(j) k}}{\partial t^{\gamma}} \frac{\partial \tilde{x}^{k}}{\partial x^{\gamma}} d t+\int_{D_{1}} \frac{\partial q^{(j) k}}{\partial t^{\gamma}} \frac{\partial \tilde{y}^{k}}{\partial x^{\gamma}} d t\right) \\
+ & \sum_{\alpha, \gamma=1}^{2} \int_{D_{1}} \frac{\partial v^{(j) \alpha}}{\partial t^{\gamma}} \frac{\partial^{2} \eta}{\partial x^{\alpha} \partial x^{\gamma}} d t \mid \\
\leq & \tilde{c} E^{1 / 2}\left(l^{(j)}, l_{0}, 1\right)+\tilde{c} \sum_{k=3}^{n}\left(\left\|x^{(j) k}\right\|_{L^{2}\left(D_{1}\right)}+\left\|y^{(j) k}\right\|_{L^{2}\left(D_{1}\right)}\right),
\end{aligned}
$$

where $\tilde{x}^{k}, \tilde{y}^{k} \eta$ and their derivatives above are evaluated at $\left(x^{(j) 1}\left(t^{1}, t^{2}\right), x^{(j) 2}\left(t^{1}, t^{2}\right)\right)$.

Since $x^{(j) \alpha}$ converges to $t^{\alpha}$ strongly in $L^{2}$ for $\alpha=1,2$, we conclude that $\frac{\partial \tilde{x}^{k}}{\partial x^{\gamma}}\left(x^{(j) 1}(t), x^{(j) 2}(t)\right), \frac{\partial \tilde{y}^{k}}{\partial x^{\gamma}}\left(x^{(j) 1}(t), x^{(j) 2}(t)\right)$ and $\frac{\partial^{2} \eta}{\partial x^{\gamma} \partial x^{\alpha}}\left(x^{(j) 1}(t), x^{(j) 2}(t)\right)$ converge strongly in $L^{2}$ to $\frac{\partial \tilde{x}^{k}}{\partial x^{\gamma}}\left(t^{1}, t^{2}\right), \frac{\partial \tilde{y}^{k}}{\partial x^{\gamma}}\left(t^{1}, t^{2}\right)$ and $\frac{\partial^{2} \eta}{\partial x^{\gamma} \partial x^{\alpha}}\left(t^{1}, t^{2}\right)$ respectively. Therefore, in view of (16) and (26), if we view $\tilde{x}^{k}, \tilde{y}^{k}, \eta$ as functions defined on $\left(t^{1}, t^{2}\right)$, we would get

$$
\begin{aligned}
& \sum_{k=3}^{n} \sum_{\gamma=1}^{2}\left(\int_{D_{1}} \frac{\partial w^{k}}{\partial t^{\gamma}} \frac{\partial \tilde{x}^{k}}{\partial t^{\gamma}} d t+\int_{D_{1}} \frac{\partial q^{k}}{\partial t^{\gamma}} \frac{\partial \tilde{y}^{k}}{\partial t^{\gamma}} d t\right) \\
& +\sum_{\alpha, \gamma=1}^{2} \int_{D_{1}} \frac{\partial v^{\alpha}}{\partial t^{\gamma}} \frac{\partial^{2} u}{\partial t^{\alpha} \partial t^{\gamma}} d t \\
& =0 .
\end{aligned}
$$


Since $\tilde{x}^{k}, \tilde{y}^{k}, \eta$ are arbitrary, we see that $w^{k}, q^{k}$ are harmonic functions for $k=3, \ldots, n$ and

$$
\sum_{\alpha, \gamma=1}^{2} \int_{D_{1}} \frac{\partial v^{\alpha}}{\partial t^{\gamma}} \frac{\partial^{2} u}{\partial t^{\alpha} \partial t^{\gamma}} d t=0
$$

for any $\eta \in C_{c}^{\infty}\left(D_{1}\right)$. To show that $\left(v^{1}, v^{2}\right)$ is the gradient of some function we notice that the map

$$
\left(t^{1}, t^{2}\right) \mapsto\left(x^{(j) 1}, x^{(j) 2}, \ldots, x^{(j) n}, v^{(j) 1}, v^{(j) 2}, q^{(j) 3}, \ldots, q^{(j) n}\right)
$$

is still isotropic for any $j$. Thus by Lemma 2.3, the weak limit as $j$ goes to infinity is still isotropic. But the weak limit is $\left(t^{1}, t^{2}, 0, \ldots, 0, v^{1}, v^{2}, q^{3}, \ldots, q^{n}\right)$. Therefore

$$
\frac{\partial v^{1}}{\partial t^{2}}-\frac{\partial v^{2}}{\partial t^{1}}=0
$$

Now combine (28) and (29), we get the existence of a biharmonic function $f: D_{1} \rightarrow \mathbb{R}$, such that $\left(v^{1}, v^{2}\right)=\nabla f$.

From standard elliptic estimates, we get

$$
\begin{aligned}
& \sup _{D_{r}}\left|u^{\alpha}-u_{0}^{\alpha}\right|^{2} \leq c r^{4} \int_{D_{1}}\left|\nabla u^{\alpha}\right|^{2} d t, \\
& \sup _{D_{r}}\left|v^{\alpha}-v_{0}^{\alpha}\right|^{2} \leq c r^{4} \int_{D_{1}}\left|\nabla v^{\alpha}\right|^{2} d t, \\
& \sup _{D_{r}}\left|w^{k}-w_{0}^{k}\right|^{2} \leq c r^{4} \int_{D_{1}}\left|\nabla w^{k}\right|^{2} d t, \\
& \sup _{D_{r}}\left|q^{k}-q_{0}^{k}\right|^{2} \leq c r^{4} \int_{D_{1}}\left|\nabla q^{k}\right|^{2} d t,
\end{aligned}
$$

where $u_{0}^{\alpha}, v_{0}^{\alpha}, w_{0}^{k}, q_{0}^{k}$ are the linear part of the Taylor expansion of each function at 0 . Using the strong $L^{2}$ convergence of $u^{(j)}, v^{(j)}, q^{(j)}, w^{(j)}$, together with (19), we get for large $j$

$$
\begin{aligned}
& \int_{D_{\theta}}\left\{\left|u^{(j)}-u_{0}\right|^{2}+\left|v^{(j)}-v_{0}\right|^{2}+\left|w^{(j)}-w_{0}\right|^{2}+\left|q^{(j)}-q_{0}\right|^{2}\right\} d t \\
\leq & 2 \int_{D_{\theta}}\left\{\left|u-u_{0}\right|^{2}+\left|v-v_{0}\right|^{2}+\left|w-w_{0}\right|^{2}+\left|q-q_{0}\right|^{2} d t\right\} \\
\leq & c \theta^{6} .
\end{aligned}
$$

Now let

$$
\tilde{l}_{0}^{(j)}=l_{0}+\sqrt{E_{j}}\left(u_{0}+\alpha^{(j)}, w_{0}+\gamma^{(j)}, v_{0}+\beta^{(j)}, q_{0}+\mu^{(j)}\right)
$$


where $\alpha^{(j)}, \ldots, \mu^{(j)}$ are defined in (17). Then

$$
\int_{D_{\theta}}\left|l^{(j)}-\tilde{l}_{0}^{(j)}\right|^{2} d t \leq c \theta^{6} E\left(l^{(j)}, l_{0}, 1\right)
$$

Claim 3. we can perturb $\tilde{l}_{0}^{(j)}$ up to order $E_{j}$ (in the $C^{1}$ norm sense) to make it a linear conformal map $l_{1}^{(j)}$ into an isotropic plane.

Proof. Notice that since $v_{0}$ is the gradient of a quadratic function in $t$, simple calculation shows that

$$
\omega\left(\tilde{l}_{0 *}^{(j)} \frac{\partial}{\partial t^{1}}, \tilde{l}_{0 *}^{(j)} \frac{\partial}{\partial t^{2}}\right)=O\left(E_{j}\right)
$$

Therefore by a linear perturbation of order $O\left(E_{j}\right)$ only in $y^{1}$ and $y^{2}$ direction, we can modify $\tilde{l}_{0}^{(j)}$ to a linear map whose image is an isotropic plane in $\mathbb{R}^{2 n}$. Also since the map $t+\sqrt{E_{j}}\left(u_{0}+\alpha^{j}\right)$ is holomorphic, we see that the Hopf differential of the perturbed linear map is of order $O\left(E_{j}\right)$ (note that we did the previous perturbation without change the $x^{1}$ and $x^{2}$ coordinates), therefore a perturbation of order $O\left(E_{j}\right)$ will make the map into a linear holomorphic map.

Therefore, we have the $L^{2}$ estimate

$$
\begin{aligned}
& \int_{D_{\theta}}\left|l^{(j)}-l_{1}^{(j)}\right|^{2} d t \\
\leq & \int_{D_{\theta}}\left|l^{(j)}-\tilde{l}_{0}^{(j)}\right|^{2} d t+\int_{D_{\theta}}\left|l_{1}^{(j)}-\tilde{l}_{0}^{(j)}\right|^{2} d t \\
\leq & c \theta^{6} E_{j}+c \theta^{2} E_{j}^{2}
\end{aligned}
$$

and the energy estimate

$$
\begin{aligned}
& \int_{D_{\theta}}\left|\nabla\left(l^{(j)}-l_{1}^{(j)}\right)\right|^{2} d t \\
\leq & \int_{D_{\theta}}\left|\nabla\left(l^{(j)}-\tilde{l}_{0}^{(j)}\right)\right|^{2} d t+\int_{D_{\theta}}\left|\nabla\left(l_{1}^{(j)}-\tilde{l}_{0}^{(j)}\right)\right|^{2} d t \\
\leq & c E_{j}+c \theta^{2} E_{j}^{2} \\
\leq & c E_{j} .
\end{aligned}
$$


Note that in (32) we can choose $\theta=\bar{\theta}$ small and $\delta_{1}$ small such that if $E_{j} \leq \delta_{1}$, then

$$
\bar{\theta}^{-4} \int_{D_{\bar{\theta}}}\left|l^{(j)}-l_{1}^{(j)}\right|^{2} d t \leq c E_{j}
$$

Now apply (a scaled version of) Proposition 4.1 (also using (34)). For any $\epsilon>0$, there exists an $\delta=\delta(\epsilon)>0$ such that if $E_{j} \leq \delta$, we get

$$
\int_{D_{\bar{\theta}}}\left|\nabla\left(l^{(j)}-l_{1}^{(j)}\right)\right|^{2} d t \leq \varepsilon \int_{D_{2 \bar{\theta}}}\left|\nabla\left(l^{(j)}-l_{1}^{(j)}\right)\right|^{2} d t+c \bar{\theta}^{-2} \int_{D_{2 \bar{\theta}}}\left|l^{(j)}-l_{1}^{(j)}\right|^{2} d t .
$$

where $c$ is an absolute constant. Combine (32) and (35) we have

$$
\begin{aligned}
& \bar{\theta}^{-2} \int_{D_{\bar{\theta}}}\left|\nabla\left(l^{(j)}-l_{1}^{(j)}\right)\right|^{2} d t \\
\leq & c \varepsilon(2 \bar{\theta})^{-2} \int_{D_{2 \bar{\theta}}}\left|\nabla\left(l^{(j)}-l_{1}^{(j)}\right)\right|^{2} d t+c \bar{\theta}^{2} E_{j}+c \bar{\theta}^{-2} E_{j}^{2} .
\end{aligned}
$$

Then choose $\bar{\theta}, \epsilon$ and $E_{j}$ small we get,

$$
\bar{\theta}^{-2} \int_{D_{\bar{\theta}}}\left|\nabla\left(l^{(j)}-l_{1}^{(j)}\right)\right|^{2} d t \leq \frac{E_{j}}{2} .
$$

(34) and (36) give a contradiction to the hypothesis that $l^{(j)}$ does not satisfy (15). This completes the proof of the lemma.

Remark 4.3. Using standard iteration argument, the excess decay lemma gives

$$
r^{-2} \int_{D_{r}(t)}\left|\nabla\left(l-l_{t, r}\right)\right|^{2} d t \leq c r^{\mu} .
$$

for any $t \in D_{1 / 2}, r \geq 0$, where $l_{t, r}$ is some linear holomorphic function into an isotropic plane. This implies that $l$ is $C^{1, \mu}$ in $D_{1 / 2}$.

\section{Higher Regularity.}

Unlike in the classical minimal surface case, the higher regularity for a $C^{1, \mu}$ isotropically stationary surface is rather a subtle problem.

In this section first we will apply a variational method to get a compactly supported $C^{1, \mu}$ solution to the equation $\operatorname{div} X=f$, where $f$ is a $C^{0, \mu}$ function with compact support in $B_{1 / 2}$ such that $\int_{B_{1}} f d t=0$. Then using this result, we construct a suitable class of $C^{1, \mu}$ isotropic variations for the 
isotropically stationary surface $\Sigma$ and get the consequent Euler-Lagrangian equation. Finally using a Schauder type estimate, we prove that $\Sigma$ is smooth.

In this section we will use $B_{r}(x)$ (simply $B_{r}$ if $\left.x=0\right)$ to denote the disk in $\mathbb{R}^{2}$ centered at $x$ with radius $r$.

\subsection{Compactly Supported Solutions to $\operatorname{div} X=f$ Using Weighted Sobolev Spaces.}

In this subsection, we will discuss the equation $\operatorname{div} X=f$ where $f$ is a $C^{0, \mu}$ function with compact support in $B_{1 / 2}$ such that $\int_{B_{1}} f d t=0$. We would like to construct a solution which is both $C^{1, \mu}$ and compactly supported in $B_{1}$. By adding an additional constraint $\frac{\partial X_{1}}{\partial x_{2}}-\frac{\partial X_{2}}{\partial x_{1}}=0$, we could get a $C^{1, \mu}$ solution from standard elliptic theory, but this solution does not generally have compact support. So we shall prove the existence by minimizing a certain functional in a weighted Sobolev space with an exponentially decay weight.

Define a weight function $\rho: B_{1} \rightarrow \mathbb{R}^{+}$by

$$
\begin{aligned}
& \rho(x) \equiv 1 \text { for } x \in B_{1 / 2}, \\
& \rho(x)=e^{-1 / d}, \text { for } x \in B_{1} \backslash B_{3 / 4}, \\
& \rho>0, \text { in the interior of } B_{1} .
\end{aligned}
$$

where $d(x)=\operatorname{dist}\left(x, \partial B_{1}\right)=1-|x|$.

Notice that any derivative of $\rho$ decays exponentially near the boundary and therefore we can extend $\rho$ smoothly by defining $\rho$ to be identically zero outside $B_{1}$.

Define the weighted $L^{2}$ norm of a function by

$$
\|u\|_{L_{\rho}^{2}\left(B_{1}\right)}:=\left(\int_{B_{1}} \rho|u|^{2} d x\right)^{1 / 2}
$$

Define the weighted $L^{2}$ space $L_{\rho}^{2}\left(B_{1}\right):=\left\{u:\|u\|_{L_{\rho}^{2}\left(B_{1}\right)}<\infty\right\}$. And define the weighted Sobolev space $H_{\rho}^{2}\left(B_{1}\right)$ to be the set of all weakly differentiable functions in $B_{1}$ such that both $u$ and $\nabla u$ are in $L_{\rho}^{2}\left(B_{1}\right) . H_{\rho}^{2}\left(B_{1}\right)$ is a Hilbert space with the inner product

$$
\langle u, v\rangle_{\rho}:=\left\langle\rho^{1 / 2} \nabla u, \rho^{1 / 2} \nabla v\right\rangle_{L^{2}}+\left\langle\rho^{1 / 2} u, \rho^{1 / 2} v\right\rangle_{L^{2}} .
$$

For a detailed discussion of weighted Sobolev spaces, see $([\mathrm{C}])$.

Now we prove a Poincaré inequality for the weighted Sobolev space. 
Lemma 5.1. Let $u \in H_{\rho}^{2}\left(B_{1}\right)$, where $\rho$ is given in (38). Then there is an absolute constant $c$ such that

$$
\inf _{\lambda \in \mathbb{R}}\|u-\lambda\|_{L_{\rho}^{2}\left(B_{1}\right)} \leq c\|\nabla u\|_{L_{\rho}^{2}\left(B_{1}\right)} .
$$

Proof. Choose $\lambda$ to be a constant such that

$$
\min \left\{\mathcal{L}^{2}\left\{x \in B_{1 / 2}: u(x) \leq \lambda\right\}, \mathcal{L}^{2}\left\{x \in B_{1 / 2}: u(x) \geq \lambda\right\}\right\} \geq 1 / 3 \mathcal{L}^{2}\left(B_{1 / 2}\right) .
$$

where $\mathcal{L}^{2}$ is the Lebesgue measure in $\mathbb{R}^{2}$

Standard Poincaré inequality(see [Si1] page 38) implies that for any $\varepsilon>0$

$$
\int_{B_{1-\varepsilon}}|u(x)-\lambda|^{2} d x \leq c \int_{B_{1-\varepsilon}}|\nabla u(x)|^{2} d x
$$

where $c$ is an absolute constant(in particular independent of $\varepsilon$ ). Define $\tilde{\rho}(d)=\rho(1-d)$. Since $\rho$ is a function of the distance $d$ to the boundary of $B_{1}$ and is monotonically decreasing for $d<1 / 8, \tilde{\rho}^{\prime}(d) \geq 0$ for $d<1 / 8$. Integrating along $\varepsilon($ using (39)), we get

$$
\int_{0}^{1 / 8} \tilde{\rho}^{\prime}(\varepsilon) \int_{B_{1-\varepsilon}}|u(x)-\lambda|^{2} d x d \varepsilon \leq c \int_{0}^{1 / 8} \tilde{\rho}^{\prime}(\varepsilon) \int_{B_{1-\varepsilon}}|\nabla u(x)|^{2} d x d \varepsilon .
$$

Therefore

$$
\begin{aligned}
& \int_{0}^{1 / 8} \tilde{\rho}^{\prime}(\varepsilon) \int_{\varepsilon<d<1 / 8}|u(x)-\lambda|^{2} d x d \varepsilon \\
\leq & c \int_{0}^{1 / 8} \tilde{\rho}^{\prime}(\varepsilon) \int_{\varepsilon<d<1 / 8}|\nabla u(x)|^{2} d x d \varepsilon+c \int_{B_{7 / 8}}|\nabla u(x)|^{2} d x .
\end{aligned}
$$

Now co-area formula gives

$$
\begin{aligned}
& \int_{0}^{1 / 8} \tilde{\rho}^{\prime}(\varepsilon) \int_{\varepsilon<d<1 / 8}|u(x)-\lambda|^{2} d x d \varepsilon \\
= & \int_{0}^{1 / 8} \tilde{\rho}^{\prime}(\varepsilon)\left(\int_{\varepsilon}^{1 / 8} \int_{\partial B_{1-s}}|\nabla d|^{-1}|u(x)-\lambda|^{2} d \sigma d s\right) d \varepsilon .
\end{aligned}
$$

Integration by parts and noticing $|\nabla d|=1$, we have(since the boundary 
terms vanish)

$$
\begin{aligned}
& \int_{0}^{1 / 8} \tilde{\rho}^{\prime}(\varepsilon) \int_{\varepsilon<d<1 / 8}|u(x)-\lambda|^{2} d x d \varepsilon \\
= & \int_{0}^{1 / 8} \int_{\partial B_{1-\varepsilon}} \rho|\nabla d|^{-1}|u(x)-\lambda|^{2} d \sigma d \varepsilon . \\
= & \int_{d<1 / 8} \rho|u(x)-\lambda|^{2} d x \\
= & \int_{B_{1}} \rho|u(x)-\lambda|^{2} d x-\int_{B_{7 / 8}} \rho|u(x)-\lambda|^{2} d x \\
\geq & \int_{B_{1}} \rho|u(x)-\lambda|^{2} d x-c \int_{B_{7 / 8}}|u(x)-\lambda|^{2} d x \\
\geq & \int_{B_{1}} \rho|u(x)-\lambda|^{2} d x-c \int_{B_{7 / 8}} \rho|\nabla u(x)|^{2} d x
\end{aligned}
$$

Similarly

$$
\int_{0}^{1 / 8} \tilde{\rho}^{\prime}(\varepsilon) \int_{\varepsilon<d<1 / 8}|\nabla u(x)|^{2} d x d \varepsilon \leq \int_{B_{1}} \rho|\nabla u(x)|^{2} d x
$$

Finally combine (40), (41) and (42), we get

$$
\int_{B_{1}} \rho|u(x)-\lambda|^{2} d x \leq c \int_{B_{1}} \rho|\nabla u(x)|^{2} d x,
$$

which completes the proof.

We now use a variational approach to construct a compactly supported $C^{1, \mu}$ solution with compact support to the equation $\operatorname{div} X=f$.

For any $f \in C^{0, \mu}\left(B_{1}\right)$ with compact support in $B_{1 / 2}$ such that $\int_{B_{1}} f d t=$ 0 , define a functional $\mathcal{F}: H_{\rho}^{2}\left(B_{1}\right) \rightarrow \mathbb{R}$ by

$$
\mathcal{F}(u):=\int_{B_{1}}\left(2^{-1} \rho|\nabla u|^{2}-f \rho u\right) .
$$

The Euler-Lagrangian equation of the critical point of $\mathcal{F}$ is

$$
\int_{B_{1}} \rho\langle\nabla u, \nabla \varphi\rangle=\int_{B_{1}} f \rho \varphi
$$

for any $\varphi \in C_{c}^{\infty}\left(B_{1}\right)$. 
Lemma 5.2. Assume $f \in C^{0, \mu}\left(B_{1}\right)$ has compact support in $B_{1 / 2}$ and $\int_{B_{1}} f d t=0$. Then there is a minimizer $u$ of the functional $\mathcal{F}$ (defined in (43)) in $H_{\rho}^{2}\left(B_{1}\right)$. Moreover $\left.\|u\|_{L_{\rho}^{2}\left(B_{1}\right.}\right) \leq c\|f\|_{L^{2}\left(B_{1}\right)}$.

Proof. Let $M_{f}:=\inf \left\{\mathcal{F}(u) \mid u \in H_{\rho}^{2}\left(B_{1}\right)\right\}$.

By choosing $u$ to be identically zero, we see $M_{f} \leq 0$. To prove the lower bound, first notice that since $\int_{B_{1}} f=0$ and $f \rho=f$, we get $\mathcal{F}(u)=$ $\mathcal{F}\left(u-u_{0}\right)$, where $u_{0}$ is any constant. So (by replacing $u$ by $u-u_{0}$ for a suitable constant $u_{0}$ ) the Poincaré inequality(Lemma 5.1) then gives $\|u\|_{L_{\rho}^{2}\left(B_{1}\right)} \leq$ $c\|\nabla u\|_{L_{\rho}^{2}\left(B_{1}\right)}$. Therefore

$$
\begin{aligned}
\mathcal{F}(u) & \geq c\|\nabla u\|_{L_{\rho}^{2}\left(B_{1}\right)}^{2}-c\|f\|_{L^{2}\left(B_{1}\right)} \cdot\|u\|_{L_{\rho}^{2}\left(B_{1}\right)} \\
& \geq c\|\nabla u\|_{L_{\rho}^{2}\left(B_{1}\right)}^{2}-c\|f\|_{L^{2}\left(B_{1}\right)} \cdot\|\nabla u\|_{L_{\rho}^{2}\left(B_{1}\right)} .
\end{aligned}
$$

The last line in the above inequality is a quadratic function of $\|\nabla u\|_{L_{\rho}^{2}\left(B_{1}\right)}$, the minimum of which is $-c\|f\|_{L^{2}\left(B_{1}\right)}^{2}$. Therefore $0 \geq M_{f} \geq-c\|f\|_{L^{2}\left(B_{1}\right)}^{2}$.

Now assume $\lim _{k \rightarrow \infty} \mathcal{F}\left(u_{k}\right)=M_{f}$ for a sequence $\left\{u_{k}\right\} \subset H_{\rho}^{2}\left(B_{1}\right)$. The paragraph above implies that (by replacing $u_{k}$ with $u_{k}-u_{k 0}$ for a suitable constant $\left.u_{k 0}\right)\left\|\nabla u_{k}\right\|_{L_{\rho}^{2}\left(B_{1}\right)} \leq c\|f\|_{L^{2}\left(B_{1}\right)}$. Then the Poincaré inequality(Lemma 5.1) tell us $\left\|u_{k}\right\|_{H_{\rho}^{2}\left(B_{1}\right)} \leq c\|f\|_{L^{2}\left(B_{1}\right)}$. Hence we may assume that $u_{k}$ converge to some $u$ weakly in $H_{\rho}^{2}\left(B_{1}\right)$ and also weakly in $L_{\rho}^{2}\left(B_{1}\right)$. Direct

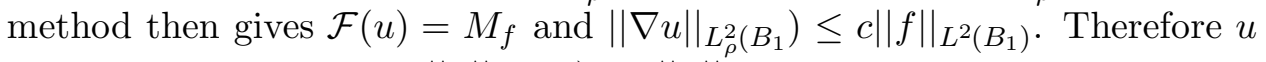
is a minimizer of $\mathcal{F}$ with $\|u\|_{L_{\rho}^{2}\left(B_{1}\right)} \leq c\|f\|_{L^{2}\left(B_{1}\right)}$.

Proposition 5.3. Let $f$ be a $C^{0, \mu}$ function in $B_{2} \subset \mathbb{R}^{2}$ such that support $f \subset B_{1 / 2}$ and $\int_{B_{2}} f=0$. Then there exists a $C^{1, \mu}$ vector field $X$ in $B_{2}$ such that

$$
\begin{aligned}
& \operatorname{div} X=f \\
& \operatorname{support}(X) \subset B_{1} \\
& \|X\|_{C^{1, \mu}\left(B_{2}\right)} \leq c\|f\|_{C^{0, \mu}\left(B_{2}\right)} .
\end{aligned}
$$

Proof. Lemma 5.2 implies that a weak solution $u$ of $\mathcal{F}$ (e.g. a minimizer) exists and satisfies $\left.\|u\|_{L_{\rho}^{2}\left(B_{1}\right.}\right) \leq c\|f\|_{L^{2}\left(B_{1}\right)} \leq c\|f\|_{C^{0, \mu}\left(B_{1}\right)}$.

Since $\rho$ is always positive in the interior of $B_{1}$, standard Schauder theory implies that $u$ is $C^{2, \mu}$ in the interior of $B_{1}$. So $u$ is a classical solution of the equation

$$
\operatorname{div}(\rho \nabla u)=f
$$


in the interior of $B_{1}$. Let $v=\rho^{1 / 2} u$, then $v \in L^{2}\left(B_{1}\right)$ and $v$ satisfies

$$
\triangle v-\frac{1}{2}\left(\rho^{-1} \triangle \rho-2^{-1} \rho^{-2}|\nabla \rho|^{2}\right) v=f .
$$

Since $\rho(x)=e^{-1 / d}$ near the boundary, the $C^{n, \mu}$ norm of $\left(\rho^{-1} \triangle \rho-\right.$ $2^{-1} \rho^{-2}|\nabla \rho|^{2}$ ) decays like $d^{-N(n)}$ near the boundary. So standard Schauder theory implies that

$$
\|v\|_{C^{2, \mu}\left(B_{1-\epsilon}\right)} \leq c \epsilon^{-N}\left(\|v\|_{L^{2}\left(B_{1}\right)}+\|f\|_{C^{0, \mu}\left(B_{1}\right)}\right) .
$$

where $N$ is a fixed positive integer.

Let $X=\rho \nabla u$, then

$$
X=\rho^{1 / 2} \nabla v-2^{-1} v \rho^{-1 / 2} \nabla \rho .
$$

So we see

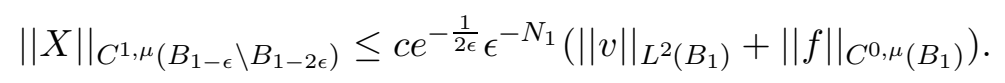

Therefore near boundary the $C^{1, \mu}$ norm of $X$ decays like $e^{-1 / 4 d}$. Thus if we extend $X$ trivially outside $B_{1}$, the new $X$ satisfies

$$
\|X\|_{C^{1, \mu}\left(B_{2}\right)} \leq c\left(\|u\|_{L_{\rho}^{2}\left(B_{1}\right)}+\|f\|_{C^{0, \mu}\left(B_{2}\right)}\right) \leq c\|f\|_{C^{0, \mu}\left(B_{2}\right)} .
$$

\subsection{Constructing Isotropic Variations.}

In this subsection we will use proposition 5.3 to construct a class of isotropic variations for an isotropic surface $\Sigma$. Notice a graphical surface $\Sigma^{*}$ over $x^{1} x^{2}$-plane given by $y^{* \alpha}, x^{* k}$ and $y^{* k}(k=3, \ldots, n$ and $\alpha=1,2)$ is isotropic if and only if

$$
\frac{\partial y^{* 1}}{\partial x^{2}}-\frac{\partial y^{* 2}}{\partial x^{1}}=\sum_{k=3}^{n} \frac{\partial x^{* k}}{\partial x^{1}} \frac{\partial y^{* k}}{\partial x^{2}}-\frac{\partial y^{* k}}{\partial x^{1}} \frac{\partial x^{* k}}{\partial x^{2}}
$$

Lemma 5.4. Assume $\Sigma$ is a $C^{1, \mu}$ isotropic surface which is graphical over $B_{2}$ in $x^{1} x^{2}$-plane. Let $\tilde{x}^{3}\left(x^{1}, x^{2}\right), \ldots, \tilde{x}^{n}\left(x^{1}, x^{2}\right), \tilde{y}^{3}\left(x^{1}, x^{2}\right), \ldots$, and $\tilde{y}^{n}\left(x^{1}, x^{2}\right)$ be smooth functions with compact support in $B_{2}$ and let $\tilde{y}^{1}$ and $\tilde{y}^{2}$ be $C^{1, \mu}$ functions with compact support in $B_{2}$. Assume

$$
\begin{aligned}
\frac{\partial \tilde{y}^{1}}{\partial x^{2}}-\frac{\partial \tilde{y}^{2}}{\partial x^{1}}= & \sum_{k=3}^{n}\left(\frac{\partial \tilde{x}^{k}}{\partial x^{1}} \frac{\partial y^{k}}{\partial x^{2}}-\frac{\partial y^{k}}{\partial x^{1}} \frac{\partial \tilde{x}^{k}}{\partial x^{2}}\right. \\
& \left.+\frac{\partial x^{k}}{\partial x^{1}} \frac{\partial \tilde{y}^{k}}{\partial x^{2}}-\frac{\partial \tilde{y}^{k}}{\partial x^{1}} \frac{\partial x^{k}}{\partial x^{2}}\right) .
\end{aligned}
$$


Then the compactly supported vector field $X$ on $\Sigma$ defined by

$$
X:=\sum_{k=3}^{n}\left(\tilde{x}^{k} \frac{\partial}{\partial x^{k}}+\tilde{y}^{k} \frac{\partial}{\partial y^{k}}\right)+\sum_{\alpha=1}^{2} \tilde{y}^{\alpha} \frac{\partial}{\partial y^{\alpha}}
$$

is isotropically integrable, i.e. there exists a family of isotropic graphical surfaces $\left\{\Sigma_{t}\right\}$ given by $y^{\alpha}\left(t, x^{1}, x^{2}\right), x^{k}\left(t, x^{1}, x^{2}\right)$ and $y^{k}\left(t, x^{1}, x^{2}\right)(k=3, \ldots, n$ and $\alpha=1,2)$ such that $\Sigma_{0}=\Sigma$ and

$$
\left.\frac{\partial y^{\alpha}(t)}{\partial t}\right|_{t=0}=\tilde{y}^{\alpha},\left.\quad \frac{\partial x^{k}(t)}{\partial t}\right|_{t=0}=\tilde{x}^{k},\left.\quad \frac{\partial y^{k}(t)}{\partial t}\right|_{t=0}=\tilde{y}^{k}
$$

for $\alpha=1,2$ and $k=3, \ldots, n$.

Proof. Let $y^{\alpha}(0)=y^{\alpha}, x^{k}(0)=x^{k}$ and $y^{k}(0)=y^{k}$. Define

$$
x^{k}(t)=x^{k}(0)+t \tilde{x}^{k}, \quad y^{k}(t)=x^{k}(0)+t \tilde{y}^{k}
$$

Then the isotropic constraint (46) for $y^{\alpha}(t), x^{k}(t)$ and $y^{k}(t)$ becomes(using the fact that $\Sigma_{0}=\Sigma$ is isotropic)

$$
\begin{aligned}
& \frac{\partial\left(y^{1}(t)-y^{1}(0)\right)}{\partial x^{2}}-\frac{\partial\left(y^{2}(t)-y^{2}(0)\right)}{\partial x^{1}} \\
= & t \sum_{k=3}^{n}\left(\frac{\partial \tilde{x}^{k}}{\partial x^{1}} \frac{\partial y^{k}}{\partial x^{2}}-\frac{\partial y^{k}}{\partial x^{1}} \frac{\partial \tilde{x}^{k}}{\partial x^{2}}+\frac{\partial x^{k}}{\partial x^{1}} \frac{\partial \tilde{y}^{k}}{\partial x^{2}}-\frac{\partial \tilde{y}^{k}}{\partial x^{1}} \frac{\partial x^{k}}{\partial x^{2}}\right) \\
+ & t^{2} \sum_{k=3}^{n}\left(-\frac{\partial \tilde{x}^{k}}{\partial x^{1}} \frac{\partial \tilde{y}^{k}}{\partial x^{2}}+\frac{\partial \tilde{y}^{k}}{\partial x^{1}} \frac{\partial \tilde{x}^{k}}{\partial x^{2}}\right) .
\end{aligned}
$$

By Proposition 5.3, there exist $C^{1, \mu}$ functions $\lambda^{1}$ and $\lambda^{2}$ with compact support in $B_{2}$ such that

$$
\frac{\partial \lambda^{1}}{\partial x^{2}}-\frac{\partial \lambda^{2}}{\partial x^{1}}=\sum_{k=3}^{n}\left(-\frac{\partial \tilde{x}^{k}}{\partial x^{1}} \frac{\partial \tilde{y}^{k}}{\partial x^{2}}+\frac{\partial \tilde{y}^{k}}{\partial x^{1}} \frac{\partial \tilde{x}^{k}}{\partial x^{2}}\right) .
$$

Now define

$$
y^{\alpha}\left(t, x^{1}, x^{2}\right):=t^{2} \lambda^{\alpha}\left(x^{1}, x^{2}\right)+t \tilde{y}^{\alpha}\left(x^{1}, x^{2}\right)+y^{\alpha}\left(0, x^{1}, x^{2}\right) .
$$

It is then easy to see that $x^{k}(t), y^{k}(t)$ and $y^{\alpha}(t)$ satisfies the isotropic constraint equation (49). So the surface $\Sigma_{t}$ is isotropic for any $t$. Moreover by differentiating (49) with respect to $t$, we see that $\tilde{x}^{k}, \tilde{y}^{k}$ and $\tilde{y}^{\alpha}$ satisfy (48) 
and (47). Therefore $X$ is isotropically integrable. This completes the proof.

Lemma 5.4 immediately implies the following Euler-Lagrangian equation for isotropically stationary surfaces:

Lemma 5.5. Assume $\Sigma$ is a $C^{1, \mu}$ isotropic surface which is graphical over $B_{2}$ in $x^{1} x^{2}$-plane. If $\Sigma$ is isotropically stationary, then

$$
\begin{aligned}
& \sum_{k=3}^{n} \int_{B_{2}} \nabla^{\Sigma} x^{k} \cdot \nabla^{\Sigma} \tilde{x}^{k} d \mu_{\Sigma}+\sum_{k=3}^{n} \int_{B_{2}} \nabla^{\Sigma} y^{k} \cdot \nabla^{\Sigma} \tilde{y}^{k} d \mu_{\Sigma} \\
& +\sum_{\alpha=1}^{2} \int_{B_{2}} \nabla^{\Sigma} y^{\alpha} \cdot \nabla^{\Sigma} \tilde{y}^{\alpha} d \mu_{\Sigma} \\
& =0,
\end{aligned}
$$

for any smooth functions $\tilde{x}^{3}\left(x^{1}, x^{2}\right), \ldots, \quad \tilde{x}^{n}\left(x^{1}, x^{2}\right), \quad \tilde{y}^{3}\left(x^{1}, x^{2}\right), \ldots$, $\tilde{y}^{n}\left(x^{1}, x^{2}\right)$ with compact support in $B_{2}$ and any $C^{1, \mu}$ functions $\tilde{y}^{1}\left(x^{1}, x^{2}\right)$ and $\tilde{y}^{2}\left(x^{1}, x^{2}\right)$ with compact support in $B_{2}$ such that (47) holds. Here $\nabla^{\Sigma}$ is the Riemannian connection of the induced metric on $\Sigma$.

Proof. By Lemma 5.4, the vector field on $\Sigma$

$$
X:=\sum_{k=3}^{n}\left(\tilde{x}^{k} \frac{\partial}{\partial x^{k}}+\tilde{y}^{k} \frac{\partial}{\partial y^{k}}\right)+\sum_{\alpha=1}^{2} \tilde{y}^{\alpha} \frac{\partial}{\partial y^{\alpha}}
$$

is isotropically integrable. Therefore apply the standard first variation formula to $X$, we get $(50)$.

\section{3. $C^{1, \mu}$ to $C^{\infty}$ and the Partial Regularity Theorem.}

Now we are in the position to prove that any $C^{1, \mu}$ isotropically stationary surface is smooth.

Proposition 5.6. Assume $\Sigma$ is a $C^{1, \mu}$ isotropic surface which is graphical over $B_{2}$ in $x^{1} x^{2}$-plane. If $\Sigma$ is isotropically stationary, then $\Sigma$ is $C^{\infty}$ in $B_{1}$.

Proof. We may assume(by scaling) that

$$
\left\|x^{k}\right\|_{C^{1, \mu}\left(B_{2}\right)} \leq \varepsilon,\left\|y^{k}\right\|_{C^{1, \mu}\left(B_{2}\right)} \leq \varepsilon,\left\|y^{\alpha}\right\|_{C^{1, \mu}\left(B_{2}\right)} \leq \varepsilon
$$


for $k=3, \ldots, n$ and $\alpha=1,2$.

In lemma 5.5 , if we let $\tilde{x}^{4}\left(x^{1}, x^{2}\right), \ldots, \tilde{x}^{n}\left(x^{1}, x^{2}\right), \tilde{y}^{3}\left(x^{1}, x^{2}\right), \ldots$, $\tilde{y}^{n}\left(x^{1}, x^{2}\right)$ be identically zero, then the Euler-Lagrangian equation for $x^{3}$ becomes

$$
\int_{B_{2}} \nabla^{\Sigma} x^{3} \cdot \nabla^{\Sigma} \tilde{x}^{3} d \mu+\sum_{\alpha=1}^{2} \int_{B_{2}} \nabla^{\Sigma} y^{\alpha} \cdot \nabla^{\Sigma} \tilde{y}^{\alpha} d \mu=0,
$$

for any smooth function $\tilde{x}^{3}\left(x^{1}, x^{2}\right)$ and $C^{1, \mu}$ functions $\tilde{y}^{1}\left(x^{1}, x^{2}\right)$ and $\tilde{y}^{2}\left(x^{1}, x^{2}\right)$ with compact support in $B_{2}$ such that

$$
\frac{\partial \tilde{y}^{1}}{\partial x^{2}}-\frac{\partial \tilde{y}^{2}}{\partial x^{1}}=\frac{\partial \tilde{x}^{3}}{\partial x^{1}} \frac{\partial y^{3}}{\partial x^{2}}-\frac{\partial y^{3}}{\partial x^{1}} \frac{\partial \tilde{x}^{3}}{\partial x^{2}} .
$$

For $f\left(x^{1}, x^{2}\right) \in C^{0, \mu}\left(B_{2}\right)$ with compact support in $B_{2}$ such that $\int_{B_{2}} f d t=$ 0 , define $\mathcal{G}(f)$ to be the set of all $\Gamma=\left(\Gamma^{1}, \Gamma^{2}\right)$ such that $\Gamma$ is $C^{1, \mu}$ vector field on $B_{2}$ with compact support in $B_{2}$ and $\frac{\partial \Gamma^{1}}{\partial x^{2}}-\frac{\partial \Gamma^{2}}{\partial x^{1}}=f$. Proposition 5.3 implies that $\mathcal{G}(f)$ is not empty.

Clearly for any $\Gamma=\left(\Gamma^{1}, \Gamma^{2}\right) \in \mathcal{G}\left(\frac{\partial \tilde{x}^{3}}{\partial x^{1}} \frac{\partial y^{3}}{\partial x^{2}}-\frac{\partial y^{3}}{\partial x^{1}} \frac{\partial \tilde{x}^{3}}{\partial x^{2}}\right)$ and any $\Pi=$ $\left(\Pi^{1}, \Pi^{2}\right) \in \mathcal{G}\left(\frac{\partial \tilde{x}^{3}}{\partial x^{1}} \frac{\partial \delta^{(h)} y^{3}}{\partial x^{2}}-\frac{\partial \delta^{(h)} y^{3}}{\partial x^{1}} \frac{\partial \tilde{x}^{3}}{\partial x^{2}}\right)$, the vector $\delta^{(h)} \Gamma-\Pi$ is a compactly supported solution to

$$
\frac{\partial \tilde{y}^{1}}{\partial x^{2}}-\frac{\partial \tilde{y}^{2}}{\partial x^{1}}=\frac{\partial \delta^{(h)} \tilde{x}^{3}}{\partial x^{1}} \frac{\partial y^{3}}{\partial x^{2}}-\frac{\partial y^{3}}{\partial x^{1}} \frac{\partial \delta^{(h)} \tilde{x}^{3}}{\partial x^{2}}
$$

where $\delta^{(h)}$ is the standard difference quotient operator. Therefore the EulerLagrangian equation (52) tells us

$$
\begin{aligned}
& \int_{\Omega} \nabla^{\Sigma} x^{3} \cdot \nabla^{\Sigma} \delta^{(h)} \tilde{x}^{3} d \mu_{\Sigma} \\
+ & \sum_{\alpha=1}^{2} \int_{\Omega} \nabla^{\Sigma} y^{\alpha} \cdot \nabla^{\Sigma}\left\{\delta^{(h)} \Gamma^{\alpha}-\Pi^{\alpha}\right\} d \mu_{\Sigma} \\
= & 0,
\end{aligned}
$$

for any $\Gamma \in \mathcal{G}\left(\frac{\partial \tilde{x}^{3}}{\partial x^{1}} \frac{\partial y^{3}}{\partial x^{2}}-\frac{\partial y^{3}}{\partial x^{1}} \frac{\partial \tilde{x}^{3}}{\partial x^{2}}\right)$ and $\Pi \in \mathcal{G}\left(\frac{\partial \tilde{x}^{3}}{\partial x^{1}} \frac{\partial \delta^{(h)} y^{3}}{\partial x^{2}}-\frac{\partial \delta^{(h)} y^{3}}{\partial x^{1}} \frac{\partial \tilde{x}^{3}}{\partial x^{2}}\right)$.

To get $C^{1, \mu}$ estimates of $\delta^{(h)} x^{3}$, we shall need the following Schauder type estimate.

Lemma 5.7. Let $a_{i j}(x), b_{i}(x), c_{i}(x), d(x), f_{i}(x)$ and $g(x)$ be $C^{0, \mu}$ functions on $B_{2}$. Assume $q_{1}(x), \ldots, q_{m}(x)$ are $C^{1, \mu}$ functions on $B_{2}$ and $p_{i k j}(x)$ are $C^{0, \mu}$ on $B_{2}$, for $i, k=1,2$ and $j=1, \ldots, m$. 
Assume $u(x) \in C^{1, \mu}\left(B_{2}\right)$ satisfies

$$
\begin{aligned}
& \sum_{i, j=1}^{2} \int_{B_{2}} a_{i j} D^{i} u D^{j} \varphi+\sum_{i=1}^{2} \int_{B_{2}} b_{i} \varphi D^{i} u+\sum_{i=1}^{2} \int_{B_{2}} c_{i} u D^{i} \varphi \\
& +\int_{B_{2}} d u \varphi \\
= & \sum_{i=1}^{2} \int_{B_{2}} f_{i} D^{i} \varphi d \mu+\int_{B_{2}} g \varphi d \mu+\sum_{j=1}^{m} \sum_{i, k=1}^{2} \int_{B_{2}} p_{i k j} D^{k} \Gamma_{j}^{i} d x .
\end{aligned}
$$

for any $\varphi \in C_{c}^{\infty}\left(B_{2}\right)$, and for any

$$
\Gamma_{j}=\left(\Gamma_{j}^{1}, \Gamma_{j}^{2}\right) \in \mathcal{G}\left(D^{1} q_{j} D^{2} \varphi-D^{2} q_{j} D^{1} \varphi\right), j=1, \ldots m
$$

We assume the ellipticity condition:

$$
\sum_{i, j=1}^{2} a_{i j}(x) \xi^{i} \xi^{j} \geq \Lambda|\xi|^{2}
$$

and

$$
\begin{aligned}
\left\|a_{i j}\right\|_{C^{0, \mu}\left(B_{2}\right)} \leq \Lambda_{1}, \quad\left\|b_{i}\right\|_{C^{0, \mu}\left(B_{2}\right)} & \leq \Lambda_{1} \\
\left\|c_{i}\right\|_{C^{0, \mu}\left(B_{2}\right)} \leq \Lambda_{1}, \quad\|d\|_{C^{0, \mu}\left(B_{2}\right)} & \leq \Lambda_{1}
\end{aligned}
$$

Then

$$
\begin{aligned}
\|u\|_{C^{1, \mu}\left(B_{1}\right)} \leq & c\left(\|u\|_{C^{0}\left(B_{2}\right)}+\|g\|_{C^{0, \mu}\left(B_{2}\right)}+\sum_{i=1}^{2}\left\|f_{i}\right\|_{C^{0, \mu}\left(B_{2}\right)}\right. \\
& \left.+\sum_{j=1}^{k} \sum_{i, k=1}^{2}\left\|p_{i k j}\right\|_{C^{0, \mu}\left(B_{2}\right)}\left\|D q_{j}\right\|_{C^{0, \mu}\left(B_{2}\right)}\right),
\end{aligned}
$$

where $c=c\left(\Lambda, \Lambda_{1}\right)$.

The proof of this lemma is a slight modification of Simon's proof of the Schauder theory in [Si3] and will be omitted here. 
Now apply lemma 5.7 to equation (55)(also using the small $C^{1, \mu}$ norm condition (51)), we get

$$
\begin{aligned}
& \left\|\delta_{i}^{(h)} x^{3}\right\|_{C^{1, \mu}\left(B_{1}\right)} \\
\leq & c\left\{\sum_{k=3}^{n}\left(\left\|\delta_{i}^{(h)} x^{k}\right\|_{C^{0}\left(B_{2}\right)}+\left\|\delta_{i}^{(h)} y^{k}\right\|_{C^{0}\left(B_{2}\right)}\right)+\sum_{\alpha=1}^{2}\left\|\delta_{i}^{(h)} y^{\alpha}\right\|_{C^{0}\left(B_{2}\right)}\right\} \\
& +c \epsilon\left\{\sum_{k=3}^{n}\left(\left\|\delta_{i}^{(h)} x^{k}\right\|_{C^{1, \mu}\left(B_{2}\right)}+\left\|\delta_{i}^{(h)} y^{k}\right\|_{C^{1, \mu}\left(B_{2}\right)}\right)\right. \\
& \left.+\sum_{\alpha=1}^{2}\left\|\delta_{i}^{(h)} y^{\alpha}\right\|_{C^{1, \mu}\left(B_{2}\right)}\right\}
\end{aligned}
$$

Similarly, the same result is true for $\delta_{i}^{(h)} x^{k}(k=4, \ldots, n)$ and $\delta_{i}^{(h)} y^{k}$ $(k=3, \ldots, n)$.

To get estimates for $\left\|\delta_{i}^{(h)} y^{\alpha}\right\|_{C^{1, \mu}\left(B_{1}\right)}$, we use a different variation. In the Euler-Lagrangian equation, choose $\tilde{x}^{k}$ and $\tilde{y}^{k}$ to be identically zero for $k=3, \ldots, n$. And let

$$
\tilde{y}^{\alpha}=\frac{\partial u}{\partial x^{\alpha}}, \quad \alpha=1,2,
$$

where $u$ is any $C^{2, \mu}$ function with compact support in $B_{1}$. Then the Euler Lagrangian equation (50) becomes

$$
\sum_{\alpha=1}^{2} \int_{\Sigma} \nabla^{\Sigma} y^{\alpha} \cdot \nabla^{\Sigma} \frac{\partial u}{\partial x^{\alpha}} d \mu_{\Sigma}=0 .
$$

Also we have the isotropic constraint equation:

$$
\frac{\partial y^{1}}{\partial x^{2}}-\frac{\partial y^{2}}{\partial x^{1}}=\sum_{k=3}^{n} \frac{\partial x^{k}}{\partial x^{1}} \frac{\partial y^{k}}{\partial x^{2}}-\frac{\partial y^{k}}{\partial x^{1}} \frac{\partial x^{k}}{\partial x^{2}}
$$

(60) and (61) form a mixed order elliptic system for $y^{1}$ and $y^{2}$. Difference quotient and standard Schauder theory for elliptic system(see [D-N]) imply 


$$
\begin{aligned}
& \left\|\delta_{i}^{(h)} y^{\alpha}\right\|_{C^{1, \mu}\left(B_{1}\right)} \\
\leq & c\left\{\sum_{k=3}^{n}\left(\left\|\delta_{i}^{(h)} x^{k}\right\|_{C^{0}\left(B_{2}\right)}+\left\|\delta_{i}^{(h)} y^{k}\right\|_{C^{0}\left(B_{2}\right)}\right)+\sum_{\alpha=1}^{2}\left\|\delta_{i}^{(h)} y^{\alpha}\right\|_{C^{0}\left(B_{2}\right)}\right\} \\
& +c \epsilon\left\{\sum_{k=3}^{n}\left(\left\|\delta_{i}^{(h)} x^{k}\right\|_{C^{1, \mu}\left(B_{2}\right)}+\left\|\delta_{i}^{(h)} y^{k}\right\|_{C^{1, \mu}\left(B_{2}\right)}\right)\right. \\
& \left.+\sum_{\alpha=1}^{2}\left\|\delta_{i}^{(h)} y^{\alpha}\right\|_{C^{1, \mu}\left(B_{2}\right)}\right\}
\end{aligned}
$$

To proceed we need an abstract lemma which is very useful in dealing with interior estimates(for a proof see [Si2]).

Lemma 5.8. Let $S$ be a real valued sub-additive function on the class of all balls in $B_{R}\left(x_{0}\right) \subset \mathbb{R}^{n}$ (i.e., $S(A) \leq \sum_{i=1}^{k} S\left(A_{i}\right)$ whenever $\left.A \subset \cup_{i=1}^{k} A_{i}\right)$. Suppose $\theta>0$ and $l \geq 0$ are given. Then there is an $\epsilon_{0}=\epsilon_{0}(\theta, l, n)>0$ such that if

$$
S\left(B_{\theta \rho}(x)\right) \leq \epsilon_{0} S\left(B_{\rho}(x)\right)+\gamma \rho^{-l}
$$

for all $B_{\rho}(x) \subset B_{R}\left(x_{0}\right)$ and for some constant $\gamma($ independent of $\rho$ and $x)$, then

$$
S\left(B_{\theta R}\left(x_{0}\right)\right) \leq C(\theta, l, n) \gamma .
$$

A scaled version of (59) and (62) gives

$$
\begin{aligned}
& \sum_{k=3}^{n}\left(\left[\delta_{i}^{(h)} x^{k}\right]_{\mu, B_{\rho / 2}(x)}+\left[\delta_{i}^{(h)} y^{k}\right]_{\mu, B_{\rho / 2}(x)}\right)+\sum_{\alpha=1}^{2}\left[\delta_{i}^{(h)} y^{\alpha}\right]_{\mu, B_{\rho / 2}}(x) \\
\leq & \epsilon\left\{\sum_{k=3}^{n}\left(\left[\delta_{i}^{(h)} x^{k}\right]_{\mu, B_{\rho}(x)}+\left[\delta_{i}^{(h)} y^{k}\right]_{\mu, B_{\rho}(x)}\right)+\sum_{\alpha=1}^{2}\left[\delta_{i}^{(h)} y^{\alpha}\right]_{\mu, B_{\rho}(x)}\right\} \\
+ & C \rho^{-2}\left\{\sum_{k=3}^{n}\left(\left\|\delta_{i}^{(h)} x^{k}\right\|_{C^{0}\left(B_{2}\right)}+\left\|\delta_{i}^{(h)} y^{k}\right\|_{C^{0}\left(B_{2}\right)}\right)\right. \\
& \left.+\sum_{\alpha=1}^{2}\left\|\delta_{i}^{(h)} y^{\alpha}\right\|_{C^{0}\left(B_{2}\right)}\right\}
\end{aligned}
$$

for any $B_{\rho}(x) \subset B_{2}$. 
Therefore applying abstract lemma 5.8 with

$$
S\left(B_{\rho}(x)\right)=\sum_{k=3}^{n}\left(\left[\delta_{i}^{(h)} x^{k}\right]_{\mu, B_{\rho}(x)}+\left[\delta_{i}^{(h)} y^{k}\right]_{\mu, B_{\rho}(x)}\right)+\sum_{\alpha=1}^{2}\left[\delta_{i}^{(h)} y^{\alpha}\right]_{\mu, B_{\rho}(x)},
$$

we get(choose $\epsilon$ small)

$$
\begin{aligned}
& \sum_{k=3}^{n}\left(\left\|\delta_{i}^{(h)} x^{k}\right\|_{C^{1, \mu}\left(B_{1}\right)}+\left\|\delta_{i}^{(h)} y^{k}\right\|_{C^{1, \mu}\left(B_{1}\right)}\right)+\sum_{\alpha=1}^{2}\left\|\delta_{i}^{(h)} y^{\alpha}\right\|_{C^{1, \mu}\left(B_{1}\right)} \\
\leq & c\left\{\sum_{k=3}^{n}\left\|\delta_{i}^{(h)} x^{k}\right\|_{C^{0}\left(B_{2}\right)}+\left\|\delta_{i}^{(h)} y^{k}\right\|_{C^{0}\left(B_{2}\right)}\right) \\
& \left.+\sum_{\alpha=1}^{2}\left\|\delta_{i}^{(h)} y^{\alpha}\right\|_{C^{0}\left(B_{2}\right)}\right\} .
\end{aligned}
$$

Let $h$ go to 0 we see that $x^{k}, y^{k}$ and $y^{\alpha}$ are $C^{2, \mu}$. Iterate, we get that they are $C^{\infty}$.

Finally combine Proposition 5.6 and Lemma 4.2(in particular remark 4.3), we have

Theorem 5.9 (Partial Regularity Theorem). Let $l: D_{2} \rightarrow \mathbb{R}^{2 n}$ be a weakly conformal, weakly isotropic map which minimizes area among all the isotropic maps with the same boundary value. Let $l_{0}$ be a linear holomorphic map into an isotropic plane. Assume $l(0)=l_{0}(0)=0$, and Area $\left(l\left(D_{1}\right)\right) \leq$ $c_{1}$. Then there is a $\varepsilon_{0} \geq 0$ depending only on $c_{1}$ such that if

$$
\int_{D_{1}}\left|\nabla\left(l-l_{0}\right)\right|^{2} d t+\int_{D_{1}}\left|l-l_{0}\right|^{2} d t \leq \varepsilon_{0}
$$

then $l$ is a smooth immersion in $D_{1 / 2}$.

\section{Tangent Cones.}

Standard technique of proving interior regularity from partial regularity involves the study of tangent cones and the use of dimension reducing argument. In the first part of this section, we construct tangent cones at each point of an isotropically area minimizing map. In the second part, we classify all the isotropically stationary cones with isolated singularities and study their stability. 
Proposition 6.1. Let $l$ be a weakly conformal, exact, isotropically stationary map from $\mathbb{R}^{2}$ to $\mathbb{R}^{2 n}$. Suppose $l(0)=0$ and $l$ is proper(in the sense that the pre-image of any compact set is compact). Also suppose that $a^{-2} \int_{\mathbb{R}^{2}} F^{(a)} \circ \tilde{l} d t$ is constant for any $a \geq 0$, where $\tilde{l}$ is a contact lifting of $l$ such that $\tilde{l}(0)=0$ and $F^{(a)}$ is defined in Section3. Then $l$ is a cone in the sense that the position vector is in the tangent plane of $l$. Moreover the contact lifting $\tilde{l}$ in $\mathbb{R}^{2 n+1}$ lies in $\{\varphi=0\}$.

Proof. First we prove that $\varphi=0$. Since $a^{-2} \int_{\mathbb{R}^{2}} F^{(a)} \circ \tilde{l} d t$ is constant for $a \geq 0$, by the monotonicity formula (12), we see that $\theta=$ constant and $\pi(\overrightarrow{\vec{P}})=0$, where $\vec{P}$ is the position vector of $l$ and $\pi$ is the orthogonal projection onto $Q$ (see (8)). Therefore $\varphi=\lambda s$, where $\lambda$ is a constant. We are going to show that $\lambda=0$. Note the modified distance function(see section 3) $r^{2}=2\left(1+\lambda^{2}\right) s$. Now fixed a ball $\tilde{B}_{a_{0}}(0)$ (defined in $\left.(6)\right)$. Let $\xi: \mathbb{R} \rightarrow \mathbb{R}$ be a concave cutoff function such that:

$$
\xi(\theta)=1, \quad \text { if } \theta \geq a_{0}^{2} 2^{-2}\left(1+\lambda^{2}\right)^{-1},
$$

and $\xi(0)=0, \xi^{\prime}(\theta)>0$ for $\theta<a_{0}^{2} 2^{-2}\left(1+\lambda^{2}\right)^{-1}$. Then we see that since $r^{2}=2^{-1}\left(1+\lambda^{2}\right) s$, the Hamiltonian function $\xi \circ s$ is identically 1 outside $\tilde{B}_{a_{0}}(0)$. Since $l$ is proper, $l^{-1}\left(B_{a_{0}}(0)\right)$ is compact in $\mathbb{R}^{2}$. Therefore the function $\xi \circ s \circ l$ is identically 1 outside a compact set in $\mathbb{R}^{2}$. Let $X_{\xi \circ s}$ be the contact vector field defined in Lemma 3.1. Then first variation gives $\int_{\Sigma} d i v_{\Sigma} X_{\xi \circ s} d \mu_{\Sigma}=0$, where $\Sigma=l\left(\mathbb{R}^{2}\right)$. Note that

$$
\operatorname{div}_{\Sigma} X_{\xi \circ s}=\xi^{\prime} \operatorname{div}_{\Sigma} X_{s}+\nabla\left(\xi^{\prime}\right) \cdot X_{s}=\xi^{\prime \prime} \nabla s \cdot X_{s},
$$

where we use the chain rule of $X_{h}$ operator and the fact that $d i v_{\Sigma} X_{s}=0$. Using the fact that $\tilde{l}$ is contact and the fact that $\varphi=\lambda s$, we get that

$$
\nabla \varphi \cdot X_{s}=\lambda \nabla s \cdot X_{s}=|\nabla \varphi|^{2} .
$$

If $\lambda \neq 0$, then we see that $\int_{\Sigma} \xi^{\prime \prime}|\nabla \varphi|^{2} d \mu_{\Sigma}=0$. Since $\xi$ is concave, $\varphi=$ constant. But since $\varphi(0)=0$ we get that $\varphi \equiv 0$ in $\tilde{l}^{-1}\left(B_{a_{0}}\right)$. The arbitrariness of $a_{0}$ gives that $\varphi \equiv 0$ in $\mathbb{R}^{2}$.

Now $\varphi \equiv 0$ implies that the position vector $\vec{P}$ is orthogonal to $\operatorname{JTl}(D)$ where $J$ is the standard complex structure in $\mathbb{R}^{2 n}$. Note as discussed in section 3 , since $l(D)$ is isotropic, the tangent space of $\mathbb{R}^{2 n}$ at the origin admits the orthogonal decomposition:

$$
T \mathbb{R}^{2 n}=T l(D) \oplus J T l(D) \oplus Q
$$


However, we already see that the orthogonal projection of $\vec{P}$ onto $Q$ is zero(since $\pi(\vec{P})=0)$. Therefore, $\vec{P} \in T l(D)$, which completes the proof.

Now we are able to construct tangent cones for an isotropically areamiminizing map. Let $l: D_{1} \rightarrow \mathbb{R}^{2 n}$ be weakly conformal, exact, isotropically area minimizing. Let $t_{0} \in D_{1}$. For any sequence $\epsilon_{j} \rightarrow 0$, define rescaled maps $l_{j}: D_{\varepsilon_{j}^{-1}} \rightarrow \mathbb{R}^{2 n}$ by

$$
l_{j}(\tau)=\delta^{-1} l\left(\varepsilon_{j} \tau+t_{0}\right)
$$

where $\delta_{j}=\sqrt{\operatorname{Area}\left(l\left(D_{2 \varepsilon}\left(t_{0}\right)\right)\right)}$ is chosen such that $\operatorname{Area}\left(l_{j}\left(D_{2}\right)\right)=1$. Clearly $l_{j}$ is still minimizing.

The same argument in Schoen and Wolfson's paper(also using Proposition 6.1) implies that (1) $\left\{l_{j}\right\}_{j=1}^{\infty}$ converges strongly in $W_{l o c}^{1,2}\left(\mathbb{R}^{2}, \mathbb{R}^{2 n}\right)$ to a weakly conformal, exact, isotropically area-minimizing map $l_{0}: \mathbb{R}^{2} \rightarrow \mathbb{R}^{2 n}$; (2) $l_{0}$ is actually a cone in the sense that the position vector lying in the tangent plane of $l$; and (3) $l_{0}$ is a proper map with $l_{0}^{-1}(\{0\})=\{0\}$.

We will henceforth call such a limiting map $l_{0}$ a tangent map of $l$ at $t_{0}$ (with respect to $\left\{\varepsilon_{j}\right\}$ ) or a tangent cone of $l$ at $t_{0}$.

The following lemma studies the case where the image of a tangent map is contained in a plane.

Lemma 6.2. Let $l_{0}(\tau): \mathbb{R}^{2} \rightarrow \mathbb{R}^{2 n}$ be a tangent map of $l$ at $t_{0}$. If the image of $l_{0}$ is contained in an isotropic plane, then (after a unitary coordinate change of $\mathbb{R}^{2 n}$ to make the image plane be the $x_{1} x_{2}$-plane) $l_{0}(\tau)=a \tau^{n}$ for some complex constant $a=\neq 0$ and some positive integer $n$. In particular $l_{0}$ is a smooth immersion away from the origin and the image of $l_{0}$ is the plane.

Proof. Because every deformation inside $x_{1} x_{2}$-plane is isotropic, $l_{0}$ is area minimizing as a map from $\mathbb{R}^{2}$ to $\mathbb{R}^{2}$. Therefore, by standard harmonic function theory, $l_{0}$ is a harmonic map, hence smooth in $\mathbb{R}^{2}$. Since $l_{0}$ is also conformal, (by possibly reversing the orientation of $\mathbb{R}^{2}$ ) we see that $l_{0}$ is holomorphic. Since $l_{0}$ is proper and $l_{0}^{-1}(\{0\})=\{0\}$, standard complex analysis theory implies $l_{0}$ is a polynomial of the form $a \tau^{n}$.

Now we study double blow-ups. 
Lemma 6.3. Let $l_{0}(\tau)$ be a tangent map of $l$ at $t_{0}$. Let $\tau_{0} \in \mathbb{R}^{2} \backslash\{0\}$. Let $l_{1}(\xi)$ be a tangent map of $l_{0}$ at $\tau_{0}$. Then the image of $l_{1}$ is a plane. In particalur, by Lemma $6.2, l_{1}$ is a smooth immersion away from the origin.

Proof. Standard blow up argument shows that the vector $x_{0}:=l_{0}\left(\tau_{0}\right)$ is actually in the tangent plane $T_{l_{1}(\xi)} l_{1}\left(\mathbb{R}^{2}\right)$ for any non-zero $\xi$. Therefore the image $l_{1}\left(\mathbb{R}^{2}\right)$ is invariant under the translation in the direction of $x_{0}$. By a unitary transformation, we may assume the direction of $x_{0}$ is the $x_{1}$-axis. Then $l_{1}\left(\mathbb{R}^{2}\right)$ is a product of $x_{1}$-axis and a one-dimensional curve $\gamma$ in $\mathbb{R}^{2 n-1}($ which is in fact a one-dimensional cone since $l_{1}\left(\mathbb{R}^{2}\right)$ is a cone). Since $l_{1}\left(\mathbb{R}^{2}\right)$ is isotropic, this curve $\gamma$ must be in $\mathbb{R}^{2 n-2}$ spanned by $x_{2}, \ldots, x_{n}, y_{2}, \ldots$, $y_{n}$. It is obvious that any deformation in $\mathbb{R}^{2 n-2}$ is isotropic with respect to $l_{1}\left(\mathbb{R}^{2}\right)$. Therefore, the fact that $l_{1}\left(\mathbb{R}^{2}\right)$ is isotropically area minimizing in $\mathbb{R}^{2 n}$ implies that $\gamma$ is absolutely length minimizing in $\mathbb{R}^{2 n-2}$. Since the only one-dimensional minimizing cone in $\mathbb{R}^{2 n-2}$ is a straight line or a straight ray, we see $\gamma$ is a line or a ray. Therefore $l_{1}\left(\mathbb{R}^{2}\right)$ is a plane or a half plane. In any case it is contained in a plane. Then by Lemma 6.2 , the image of $l_{1}$ is a plane.

In the rest of this section we study two-dimensional isotropically stationary cones which are smooth away from origin.

First derive a geometric version of the Euler-Lagrangian equation for isotropically stationary surfaces.

Theorem 6.4. Suppose $\Sigma^{2} \subset \mathbb{R}^{2 n}$ is an isotropically stationary surface with mean curvature vector $H$. Then

$$
\begin{aligned}
& \delta(H\rfloor \omega)=0, \\
& d(H\rfloor \omega)=2 \beta, \\
& H \in J T \Sigma,
\end{aligned}
$$

where $\beta$ is $(0,2)$-tensor on $\Sigma$ defined by $\beta(X, Y)=\sum_{j=1}^{2}\left\langle\nabla_{e_{j}}^{\perp} Y, J \nabla_{e_{j}}^{\perp} X\right\rangle$, with $\left\{e_{j}\right\}_{j=1}^{2}$ being an orthonormal basis for $T \Sigma$.

Proof. To get the second equation, use Cartan's formula, we have

$$
d(H\rfloor \omega)(X, Y)=\left\langle\nabla_{X} H, J Y\right\rangle-\left\langle\nabla_{Y} H, J X\right\rangle .
$$


Let $\left\{e_{j}\right\}_{j=1}^{2}$ is an orthonormal basis for $T \Sigma$. Then $H=\sum_{j=1}^{2} \nabla_{e_{j}}^{\perp} e_{j}$. Therefore

$$
\left\langle\nabla_{X} H, J Y\right\rangle=\sum_{j=1}^{2}\left\langle\nabla_{X} \nabla_{e_{j}}^{\perp} e_{j}, J Y\right\rangle=\sum_{j=1}^{2}\left\langle\nabla_{X}^{\perp} \nabla_{e_{j}}^{\perp} e_{j}, J Y\right\rangle
$$

where we used the fact that $J Y$ is in $(T \Sigma)^{\perp}$ since $\Sigma$ is isotropic.

Assume the basis $\left\{e_{j}\right\}_{j=1}^{2}$ is normal at one point. Then using Codazzi equation(see [D]) and the fact that $\left\langle\nabla \frac{\perp}{X} Y, J Z\right\rangle$ is fully symmetric for $X, Y, Z$ in $T \Sigma$, we get

$$
d(H\rfloor \omega)(X, Y)=\sum_{j=1}^{k} 2\left\langle\nabla_{e_{j}}^{\perp} Y, J \nabla_{e_{j}}^{\perp} X\right\rangle
$$

This completes the proof of the second equation.

To get the third equation, note that since $\Sigma$ is isotropically stationary, $\int_{\Sigma}\langle H, X\rangle d \mu_{\Sigma}=0$ for any isotropic vector field with compact support. Since any $X \in(J T \Sigma)^{\perp}$ satisfies $\left.(X\rfloor \omega\right)\left.\right|_{\Sigma}=0$, it gives rise to a (local) isotropic variation. This implies that $H \in J T \Sigma$.

First variation also implies $\int_{\Sigma}\langle H, J \nabla f\rangle d \mu_{\Sigma}=0$ for any $f: M \rightarrow \mathbb{R}$ which has compact support in $\Sigma$. But since $H \in J T \Sigma$, we get that $\int_{\Sigma}\left\langle H, J \nabla^{\Sigma} f\right\rangle d \mu_{\Sigma}=0$, where $\nabla^{\Sigma} f$ is the projection of $\nabla f$ onto $T \Sigma$. This implies $\left.\int_{\Sigma}\langle\delta(H\rfloor \omega), f\right\rangle d \mu_{\Sigma}=0$. The arbitrariness of $f$ implies $\left.\delta(H\rfloor \omega\right)=0$.

Remark 6.5. The theorem above is true for any isotropically stationary submanfold $\Sigma^{k}$ in a Kähler manifold $\left(N^{2 n}, g, J\right)$, only with the second equation $d(H\rfloor \omega)=2 \beta$ replaced by $d(H\rfloor \omega)=2 \beta+2$ ric $c_{\Sigma}$, where $\operatorname{ric}_{\Sigma}(X, Y):=\operatorname{Ric}_{\Sigma}(X, J Y)$ for $X, Y$ in $T \Sigma$.

Now we can classify all isotroically stationary cones in $\mathbb{R}^{2 n}$

Theorem 6.6. Let $\Sigma$ be an isotropic cone over the origin ( in the sense that $\eta_{\lambda \#} \Sigma=\Sigma$ for any $\lambda>0$, where $\eta_{\lambda}: \mathbb{R}^{2 n} \rightarrow \mathbb{R}^{2 n}$ is the homothety map defined by $\left.\eta_{\lambda} x=x / \lambda\right)$. Assume also that $\Sigma$ is smooth away from origin and is isotropically stationary in $\mathbb{R}^{2 n}$. Then (up to a unitary transformation), the intersection of $\Sigma$ with $S^{2 n-1}$ is a curve $\gamma:[0,2 \pi] \rightarrow \mathbb{R}^{2 n}$ parametrized 
by(using complex coordinate in $\mathbb{R}^{2 n}$ )

$$
\begin{aligned}
& z_{1}(s)=\sqrt{\frac{q}{p+q}} e^{\sqrt{-1} p s} \\
& z_{2}(s)=\sqrt{\frac{p}{p+q}} e^{-\sqrt{-1} q s} \\
& z_{3}(s)=0, \ldots, z_{n}(s)=0 .
\end{aligned}
$$

for some positive integers $p$ and $q$.

Proof. Let $\gamma(\theta):[0, L] \rightarrow S^{2 n-1}$ be parametrized by arclength(i.e, $\left\|\gamma^{\prime}(\theta)\right\|=$ 1 for any $\theta$ ). Then the cone $\Sigma$ can be parametrized as

$$
\sigma(t, \theta)=t \gamma(\theta), \quad t \in(0, \infty), \theta \in[0, L]
$$

Note that an orthonormal basis for tangent planes of $\Sigma$ is given by $e_{1}=$ $\sigma_{*} \frac{\partial}{\partial t}=\gamma(\theta)$ and $e_{2}=\frac{\sigma_{*} \frac{\partial}{\partial \theta}}{\left\|\sigma_{*} \frac{\partial}{\partial \theta}\right\|}=\dot{\gamma}(\theta)$. Then the mean curvature vector of $\Sigma$ is given by $H=\nabla_{e_{1}}^{\perp} e_{1}+\nabla_{e_{2}}^{\perp} e_{2}=t^{-1}(\ddot{\gamma}(\theta))^{\perp}$. Calculation using Theorem 6.4 gives

$$
\ddot{\gamma}-b_{0} J \dot{\gamma}+\gamma=0,
$$

for some real constant $b_{0}$. Let $\gamma(\theta)=\left(z_{1}(\theta), \ldots, z_{n}(\theta)\right)$ where $\left(z_{1}, \ldots, z_{n}\right)$ is the complex coordinate in $\mathbb{R}^{2 n}$. Then (63) gives us

$$
\ddot{z}_{k}-\sqrt{-1} b_{0} \dot{z}_{k}+z_{k}=0, \quad \text { for } k=1, \ldots, n \text {. }
$$

Solve it, we get

$$
z_{k}=c_{k} e^{\sqrt{-1} \lambda_{1} \theta}+d_{k} e^{\sqrt{-1} \lambda_{2} \theta}
$$

where $c_{k}$ and $d_{k}$ are complex constants and

$$
\lambda_{1}=\frac{b_{0}+\sqrt{b_{0}^{2}+4}}{2}, \quad \lambda_{2}=\frac{b_{0}-\sqrt{b_{0}^{2}+4}}{2} .
$$

Moreover since $\langle\gamma, \gamma\rangle=1,\langle\dot{\gamma}, \dot{\gamma}\rangle=1$ and $\langle\dot{\gamma}, J \gamma\rangle=0$, we have

$$
\left\{\begin{array}{l}
\sum_{k=1}^{n} c_{k} \bar{c}_{k}=\frac{\lambda_{2}}{\lambda_{2}-\lambda_{1}}, \\
\sum_{k=1}^{n} d_{k} \bar{d}_{k}=\frac{\lambda_{1}}{\lambda_{1}-\lambda_{2}}, \\
\sum_{k=1}^{n} c_{k} \bar{d}_{k}=0 .
\end{array}\right.
$$


Therefore by a unitary transformation we may assume $c_{1}=\sqrt{\frac{\lambda_{2}}{\lambda_{2}-\lambda_{1}}}, c_{2}=$ $\ldots=c_{n}=0$ and $d_{1}=0, d_{2}=\sqrt{\frac{\lambda_{1}}{\lambda_{1}-\lambda_{2}}}, d_{3}=\ldots=d_{n}=0$. Thus

$$
\left[\begin{array}{c}
z_{1}(\theta) \\
z_{2}(\theta) \\
\vdots \\
z_{n}(\theta)
\end{array}\right]=e^{\sqrt{-1} \lambda_{1} \theta}\left[\begin{array}{c}
\sqrt{\frac{\lambda_{2}}{\lambda_{2}-\lambda_{1}}} \\
0 \\
\vdots \\
0
\end{array}\right]+e^{\sqrt{-1} \lambda_{2} \theta}\left[\begin{array}{c}
0 \\
\sqrt{\frac{\lambda_{1}}{\lambda_{1}-\lambda_{2}}} \\
\vdots \\
0
\end{array}\right]
$$

Since $\gamma$ is closed, we get (note $\lambda_{1}>0$ and $\lambda_{2}<0$ )

$$
\lambda_{1} L=2 \pi p, \quad \lambda_{2} L=-2 \pi q,
$$

for some positive integers $q$ and $p$, where $L$ is the length of $\gamma$. Now use the fact that $\lambda_{1} \lambda_{2}=-1$ we deduce

$$
\lambda_{1}=\sqrt{\frac{p}{q}}, \quad \lambda_{2}=-\sqrt{\frac{q}{p}} .
$$

The theorem then follows by a reparametrization of the curve using $s=$ $2 \pi \theta / L$.

We can see that these isotropic cones are exactly those Lagrangian cones described in Schoen and Wolfson's paper [S-W]. By applying the second variational formula, they have proven

Proposition 6.7 (Schoen-Wolfson). The cones with $|p-q|>1$ are strictly unstable in $\mathbb{R}^{4}$ for any Hamiltonian variation fixing a neighborhood of the cone vertex. And cones with $|p-q|=1$ are strictly stable in $\mathbb{R}^{4}$ for any Hamiltonian variation fixing a neighborhood of the cone vertex.

And the multiply covered cones are strictly unstable for any $p$ and $q$.

Therefore those cones with $|p-q|>1$ are automatically strictly unstable in $\mathbb{R}^{2 n}$. And by a careful study of the second variation, we actually get that cones with $|p-q|=1$ are also strictly stable in $\mathbb{R}^{2 n}$.

Proposition 6.8. Those cones with $|p-q|=1$ are strictly stable in $\mathbb{R}^{2 n}$ for any Hamiltonian variation fixing a neighborhood of the cone vertex.

Proof. Let $f: \mathbb{R}^{2 n} \rightarrow \mathbb{R}$ be any smooth function which is supported in $B_{R}(0) \backslash B_{\varepsilon}(0)$. Let $Z=J \nabla f$ be the Hamiltonian vector field. Therefore 
$\left.Z\right|_{\Sigma}$ is of the form

$$
J \nabla h+\sum_{k=3}^{n}\left(f_{k} \frac{\partial}{\partial x^{k}}+g_{k} \frac{\partial}{\partial y^{k}}\right)
$$

where $h, f_{k}$ and $g_{k}$ are arbitrary function of $x^{1}, x^{2}, y^{1}$ and $y^{2}$. Then using second variational formula(see $[\mathrm{S}-\mathrm{W}]$ ) we see

$$
\delta^{2}|\Sigma|\left(V_{J \nabla f}\right)=\delta^{2}|\Sigma|\left(V_{J \nabla h}\right)+\delta^{2}|\Sigma|\left(V_{W}\right),
$$

where $W=\sum_{k=3}^{n} f_{k} \frac{\partial}{\partial x^{k}}+g_{k} \frac{\partial}{\partial y^{k}}$ is in $\mathbb{R}^{2 n-4}$ and where $\delta^{2}|\Sigma|\left(V_{Z}\right)$ denotes the second variation with respect to the vector field $Z$. To prove that cones with $|p-q|=1$ are strictly stable, it suffices( since those cones are already strictly stable in $\mathbb{R}^{4}$ for any Hamiltonian variation) to show that $\delta^{2}|\Sigma|\left(V_{W}\right)>0$ for any vector field $W$ in $\mathbb{R}^{2 n-4}$. A direct computation shows that

$$
=\int_{[\epsilon, R] \times[0, L]}^{\delta^{2}|\Sigma|\left(V_{W}\right)}\left\{\left|\frac{\partial W}{\partial t}\right|^{2}+t^{-2}\left|\frac{\partial W}{\partial \theta}\right|^{2}+b_{0} t^{-2}\left\langle W, J \frac{\partial W}{\partial \theta}\right\rangle\right\} t d t d \theta
$$

where we assume $\gamma(\theta)$ is parametrized by arclength and $L$ is the length of the curve.

For notational simplicity, we only prove the case where $n=3$ (the general case follows from the same argument). Assume $W=f_{3} \frac{\partial}{\partial x^{3}}+g_{3} \frac{\partial}{\partial y^{3}}$. Let $z=x^{3}+\sqrt{-1} y^{3}$.

By the $L^{2}$ orthogonal decomposition of $W$, it suffices to prove the case where $W=f(t) e^{\sqrt{-1} 2 \pi k \theta / L}$ where $k$ is an integer. Straightforward calculation shows that

$$
\begin{aligned}
& \delta^{2}|\Sigma|\left(V_{W}\right) \\
= & L \int_{\varepsilon}^{R} t\left|f^{\prime}(t)\right|^{2} d t+L \frac{2 \pi k}{L}\left(\frac{2 \pi k}{L}-b_{0}\right) \int_{\varepsilon}^{R} t^{-2}|f(t)|^{2} d t .
\end{aligned}
$$

Note that ( without loss of generality assume $p>q$ )

$$
b_{0}=\sqrt{\frac{p}{q}}-\sqrt{\frac{q}{p}}, \quad L=2 \pi \sqrt{p q} .
$$

Since $p-q=1, \frac{2 \pi k}{L}\left(\frac{2 \pi k}{L}-b_{0}\right)>0$ for any integer $k \neq 0$. This implies that for any integer $k$

$$
\delta^{2}|\Sigma|\left(V_{W}\right)>0
$$

for $W=f(t) e^{\sqrt{-1} 2 \pi k \theta / L}$. 


\section{Interior Regularity Theorem.}

In section 2, we showed that the weak solution to the isotropically constrained Plateau problem exists. In this section we will prove that this weak solution is smooth away from a finite set.

For a map $l: \Omega \rightarrow \mathbb{R}^{2 n}$. Define regular points to be points in $\Omega$ at which $l$ is a smooth immersion. We denote the set of all regular points as $\operatorname{Reg}(l)$. And define the singular set $\operatorname{Sing}(l)=\Omega \backslash \operatorname{Reg}(l)$.

The following proposition says that singularities converge to a singularity.

Proposition 7.1. Let $l^{(k)}: D_{2} \rightarrow \mathbb{R}^{2 n}$ be a sequence of weakly conformal, weakly exact and isotropically area minimizing maps. Assume $\left\{l^{(k)}\right\}_{k=1}^{\infty}$ converges strongly in $W_{l o c}^{1,2}\left(D_{2}, \mathbb{R}^{2 n}\right)$ to a weakly conformal, exact and isotropically area minimizing map $l$. Let $t^{(k)} \in \operatorname{Sing}\left(l^{(k)}\right) \cap D_{1}$. Suppose $\lim _{j \rightarrow \infty} t^{(k)}=t_{0}$. Then $t_{0} \in \operatorname{Sing}(l)$.

Proof. Without loss of generality, assume $t_{0}=0$. Assume 0 is a regular point of $l$. Let $\varepsilon_{j}$ be a sequence going to 0 . Define the corresponding tangent map $\varphi$ of $l$ at 0 , as the limit of $l_{j}(\tau)=\delta_{j}^{-1} l\left(\varepsilon_{j} \tau\right)$. For each $l^{(k)}$ define $l_{j}^{(k)}(\tau)=\delta_{j}^{-1} l^{(k)}\left(\varepsilon_{j} \tau\right)$. Now for a fixed $j$, since $\left\{l^{(k)}\right\}_{k=1}^{\infty}$ converges to $l$, we have an $\alpha_{j}$ such that

$$
\left\|l_{j}^{\left(\alpha_{j}\right)}-l_{j}\right\|_{W^{1,2}\left(D_{1}\right)} \leq \frac{1}{j}
$$

and since $\left\{t^{(k)}\right\}_{k=1}^{\infty}$ goes to 0 , we might also assume

$$
\left|\frac{t^{\left(\alpha_{j}\right)}}{\varepsilon_{j}}\right| \leq \frac{1}{j} .
$$

Therefore, we easily get that

$$
\lim _{j \rightarrow \infty} l_{j}^{\left(\alpha_{j}\right)}=\varphi
$$

in $W^{1,2}\left(D_{1}, \mathbb{R}^{2 n}\right)$.

Since 0 is regular for $l$, we see that $\varphi$ is a linear conformal map into a isotropic plane. Therefore, we can apply our local regularity theorem(Theorem 5.9) to get a constant $\theta$ such that for sufficient large $j, l_{j}^{\left(\alpha_{j}\right)}$ is smooth immersion in $D_{\theta}$. But note that $\frac{t^{\left(\alpha_{j}\right)}}{\varepsilon_{j}}$ is a singularity of $l_{j}^{\left(\alpha_{j}\right)}$, and $\frac{t^{\left(\alpha_{j}\right)}}{\varepsilon_{j}} \rightarrow 0$. A contradiction. 
An immediate consequence of the above proposition is the following result.

Proposition 7.2. Let $l: D_{1} \rightarrow \mathbb{R}^{2 n}$ be weakly conformal, weakly exact and isotropically area minimizing map. Let $t_{0} \in D_{1}$. If every tangent cone of $l$ at $t_{0}$ is a smooth immersion away from the origin, then $l$ is Hölder continuous in $D_{1}$ and is a smooth immersion in $D_{r}\left(t_{0}\right) \backslash\left\{t_{0}\right\}$ for some $r>0$.

Proof. Without loss of generality, assume $t_{0}=0$. If $l$ is not smooth immersion in any deleted neighborhood of 0 , then we have a sequence of points $\left\{t_{j}\right\}_{j=1}^{\infty} \subset$ $\operatorname{Sing}(l)$ which converges to 0 . Let $\varepsilon_{j}=\left|t_{j}\right|$. Let $l_{0}$ be the tangent cone at 0 with respect to $\left\{\varepsilon_{j}\right\}_{j=1}^{\infty}$ with the corresponding rescaled sequence $\left\{l_{j}\right\}_{j=1}^{\infty}$. Clearly $\tau_{j}=t_{j} / \varepsilon_{j}$ is a singularity of $l_{j}$. Since $\left|\tau_{j}\right|=1$, we might assume( by passing to a subsequence) that $\left\{\tau_{j}\right\}_{j=1}^{\infty}$ converges to a point $\tau$ in the unit circle $S^{1}$. By Proposition $7.1 \tau$ should be a singularity of the limiting map $l_{0}$. But by hypothesis, every tangent map at 0 is regular away from the origin. A contradiction.

Now we are in the position to prove the main regularity theorem.

Theorem 7.3 (Interior Regularity Theorem). Let $l: D_{2} \rightarrow \mathbb{R}^{2 n}$ be a weakly conformal, weakly isotropic map which minimizes area among isotropic maps with the same boundary value. There is a finite subset $S$ of $D_{1}$ such that $l$ is a smooth immersion on $D_{1} \backslash S$.

Proof. Let $\Omega$ be the set of points in $D_{1}$ such that the image of every tangent map is a plane. Let $t_{0} \in \Omega$. Then Lemma 6.2 says that every tangent cone of $t_{0}$ is a smooth immersion away from the origin. Therefore, by Proposition $7.2, l$ is a smooth immersion in a deleted neighborhood of $t_{0}$.

Let $B=D_{1} \backslash \Omega$. For a point $t_{1} \in B$, let $l_{0}$ be a tangent cone at $t_{1}$ whose image is not a plane. Let $\tau_{0} \in \mathbb{R}^{2}$ be any point away from the origin. Lemma 6.3 then says that any tangent cone of $l_{0}$ at $\tau_{0}$ is a smooth immersion away from the origin. Therefore by Proposition $7.2, l_{0}$ is smooth immersion in a deleted neighborhood of any point away from the origin. This implies that the singular set of $l_{0}$ is discrete. Then the fact that $l_{0}$ is a geometric cone implies that $l_{0}$ is smooth away from the origin. Therefore, by the classification of isotropically stationary cones(see Theorem 6.6) we see that (by Proposition 6.7) the cone $C=l_{0}\left(\mathbb{R}^{2}\right)$ is conformal equivalent to the complex 
plane $\mathbb{R}^{2}$ via a map $\xi: C \rightarrow \mathbb{R}^{2}$. Thus $\xi \circ l_{0}$ is a weakly conformal, isotropically minimizing, proper map with only one zero. Therefore by Lemma 6.2 , we see $\xi \circ l_{0}$ is smooth immersion away from the origin. This implies that $l_{0}$ itself is a smooth immersion away from origin. Thus Proposition 7.2 gives that $l$ is a smooth immersion in a deleted neighborhood of $t_{0}$.

So combine the above two arguments, we see that $\operatorname{Sing}(l)$ is discrete (hence finite) in $D_{1}$.

Now finally combine Theorem 2.9 and Theorem 7.3 we have the following regularity result of the solution to the isotropically constrained Plateau problem:

Theorem 7.4. Let $D_{1}$ be the unit disk in $\mathbb{R}^{2 n}$. Let $\Gamma$ be a closed piecewise $C^{1}$ Jordan curve in $\mathbb{R}^{2 n}$ such that $\int_{\Gamma}(x d y-y d x)=0$. Let

$$
\begin{aligned}
\mathcal{X}_{\Gamma, I}=\{l \in & W_{I}^{1,2}\left(D_{1}, \mathbb{R}^{2 n}\right): \\
& \left.\left.l\right|_{\partial D_{1}} \text { is continous and is a monotone map onto } \Gamma\right\} .
\end{aligned}
$$

Then $\mathcal{X}_{\Gamma, I}$ is not empty and there exists an area minimizer $l_{0}$ among $\mathcal{X}_{\Gamma, I}$. Moreover, $l_{0}$ is Hölder continuous in $D_{1}$ and is smooth immersion in the interior of $D_{1}$ with possibly isolated singularities.

\section{References.}

[Al] Allcock,D., An isoperimetric inequality for the Heisenberg groups, GAFA, 8(1998) 219-233.

[C] Corvino, J., Scalar curvature deformation and applications to general relativity, Ph.D thesis, Stanford University, 2000.

[D] Do Carmo, Manfredo,P.,Riemannian Geometry, Birkhäuser, 1992.

[D-N] Douglis,A., Nirenberg,L., Interior estimates for elliptic systems of equations, Comm. Pure Appl. Math. 8 (1955), 503-538.

[Fo] Folland,G., Introduction to partial differential equations, Princeton University Press, 1976.

[G-T] Gilbarg,D.,and Trudinger,N.S., Elliptic Partial Differential Equations of Second Order, 2nd edition, Springer,1998.

[Gr] Gromov,M., Carnot-Caratheodory spaces seen from within, Progress in Mathematics 144(1996), 79-323. 
[H-L] Harvey,R., Lawson,H.B.Jr, Calibrated Geometries, Acta. Math,148 (1982),47-157.

[La] Lawson,H.B., Lectures on minimal submanifolds, Vol 1, Publish or Perish, Inc, Berkeley, 1980.

[Mi] Minicozzi,W.,Ph.D. thesis, Stanford University.

[M-S] Macduff,D., Salamon,D.,Introduction to symplectic topology, second edition, Oxford University Press, 1998.

[Mo] Morrey,C., Multiple Integrals in the Calculus of Variations, SpringerVerlag, New York, 1966.

[Oh] Oh,Y-G, Volume minimization of Lagrangian submanifolds under Hamiltonian deformations, Math.Z., 212 (1993), 175-192.

[S-W] Schoen,R., and Wolfson,J., Minimizing area among Lagrangian surfaces: the mapping problem, Journal of Differential Geometry, $\mathbf{5 8}(2001), 1-87$.

[Si1] Simon,L.,Lectures on Geometric Measure Theory, proceedings of the Center for Mathematical Analysis, Australian National University, 1984.

[Si2] Simon,L., Theorems on regularity and singularity of energy minimizing maps, Birkhuser Verlag, c1996.

[Si3] Simon,L., Schauder estimates by scaling, Calc. Var. Partial Differential Equations 5(1997), no. 5, 391-407.

Dept of Mathematics

HARVARD UNIV. MA 02138 USA

qiu@math.harvard.edu

RECEIVED November 28, 2001. 